\title{
Aircraft and passenger recovery during an aircraft's unexpected unavailability
}

\author{
Yücel Naz Yetimoğlu ${ }^{\text {a }}$, M. Selim Aktürk ${ }^{\text {b,* }}$ \\ ${ }^{a}$ Booth School of Business, The University of Chicago, Chicago, IL, 60637, USA \\ ${ }^{\mathrm{b}}$ Department of Industrial Engineering, Bilkent University, Ankara, 06800, Turkey
}

\section{A R T I C L E I N F O}

\section{Keywords:}

Disruption management

Integrated aircraft and passenger recovery

\begin{abstract}
A B S T R A C T
Airlines design their initial schedules under the assumption that all resources will be available on time and flights will operate as planned. However, some disruptions occur due to mechanical failures and unexpected delays of maintenance, making the aircraft unavailable for a certain period of time. These deviations from the initial plan result in high operational costs in addition to the serious inconveniences experienced by passengers. In order to handle aircraft and passenger recovery problems simultaneously, we work on integrated networks at which aircraft routings and passenger itineraries are superimposed. Consequently, we could calculate the actual profit and cancellation cost by evaluating each passenger itinerary while considering the seat capacity limitations. In our computational results, we use a daily schedule of a major U.S. airline and clearly demonstrate that there is an optimal trade-off between operating and passenger-related costs.
\end{abstract}

\section{Introduction}

Air transportation fosters mobility and provides a crucial level of connectivity on a global scale. As being a convenient transportation option, the number of passengers who utilize air travel maintains positive growth rates. Due to the high and increasing demand, competition between airlines has intensified in recent years. Each airline aims to capture a greater portion of the market demand and tries to use its resources in the best possible way to enhance the profitability of its operations. Due to the competitive nature of the industry, airlines design tight schedules and utilize their fleet of aircraft as much as possible to generate more profit. They assume that all required resources will be available on time and flights will operate as planned. However, some irregularities might occur during actual operations and original schedules can easily be disrupted. Bratu and Barnhart (2006) discussed main sources of disruption and highlighted the effect of resource shortages including the absence of aircraft. To exemplify, mechanical failures can be observed and aircraft might need to undergo unscheduled maintenance making the aircraft unavailable for a period of time. Such irregularities prevent airlines from continuing their original schedules.

Among airline resources, aircraft is the scarcest one and hence even minor perturbations in aircraft schedules might result in severe disruptions. Flight delays and cancellations become inevitable depending on the unavailability duration. A recent example is that after two fatal crashes of Boeing 737 Max aircraft in October 2018 and March 2019, aviation authorities grounded the entire fleet of that type as a safety precaution. The fleet is not permitted to fly until further notice. This capacity loss critically disrupted initially generated flight schedules of airlines. In particular, Southwest Airlines, Air Canada and American Airlines face the most disruption as they have the highest number of Boeing 737 Max in their fleets (U.S. Department of Transportation, Federal Aviation Administration, 2019). In another recent example, a major US airline has faced unscheduled maintenance disruptions arising from contract negotiations. Unscheduled maintenance can occur anytime and by definition, it is unforeseen. Besides, it is also possible that planned inspections can take longer than anticipated, creating a need for recovery actions. Unless real-time and efficient recovery solutions are readily available, aircraft shortages may result in substantial loss of profit and decrease level of service for airlines in such scenarios.

Re-timing of departures, cancellation of certain flights and swapping aircraft are the most common recovery actions in cases of disruptions. Airline Operations Control Centers (AOCC) are responsible for taking such actions and managing operations of aircraft, crew and passengers centrally. Interdependencies between different entity types make it even more challenging to generate a comprehensive recovery solution. The complexity of networks as well as the high operational costs necessitates

\footnotetext{
* Corresponding author.

E-mail address: akturk@bilkent.edu.tr (M. Selim Aktürk).
} 
the use of optimization tools in this large-scale system. Due to the quick response requirements and large number of variables, proposing a single formulation for the integrated airline recovery problem is not easy. Instead, it is a common practice to adopt sequential approaches which recover aircraft, crew and passenger schedules respectively. However, without using a fully integrated approach, it is not possible to find the optimal trade-off between operating and passenger-related costs. Besides, as passenger recovery corresponds to the final stage in a sequential framework, passengers might experience large impacts of re-timing and cancellation decisions which are made in advance. Therefore, airlines should place a special emphasis on passenger convenience in order to maintain a competitive edge in the market and to increase profit in the long run.

In this study, our aim is to develop a framework to help airlines in handling schedule perturbations specifically caused by the unavailability of aircraft. We address aircraft and passenger recovery problems simultaneously instead of adopting a sequential schedule recovery framework as commonly done in literature. We utilize exchange of aircraft and cruise time controllability along with the cancellation option and propose a novel math-heuristic algorithm. Another point is that we work on integrated networks at which aircraft routings and passenger itineraries are superimposed. Considering the interdependence among entities, cancellation or re-timing decisions which are made for flight legs directly affect itineraries. As a significant contribution, we differentiate between direct and connecting passengers, prioritize affected ones and make individual passenger itinerary based recovery decisions under seat capacity restrictions. This allows us to use limited resources, in our case available seats, in a more effective way. Lastly, due to the high level of competition in airline industry, it is significant to quantify the passenger-related disruption costs and measure the inconvenience if a flight is cancelled, and hence we provide actual cancellation cost formulations by evaluating each itinerary separately.

In sum, the main contributions of this study can be listed as follows:

- We consider the integrated aircraft and passenger recovery problem within the context of aircraft unavailability period.

- To the best of our knowledge, this is the first study in which the cruise speed control is included as a decision variable in an integrated aircraft and passenger recovery problem.

- We devise a novel math-heuristic algorithm which makes individual passenger itinerary based recovery decisions under seat capacity limitations by differentiating between passengers who are on the same flight.

- We propose actual profit and cancellation cost formulations depending on passenger itineraries while evaluating a critical tradeoff between the fuel consumption and its impact on the passenger recovery related costs.

The remainder of the paper is organized as follows. Section 2 reviews existing studies on disruption management, especially the studies on passenger and aircraft recovery. In Section 3, we briefly describe the framework of the problem and the math-heuristic algorithm. First, we give the mathematical model for aircraft recovery under Phase I. Then we explain the steps of passenger recovery and integrated search algorithms in detail. In Section 4, we provide a numerical example to highlight the important steps of the proposed algorithm. Section 5 presents recovery strategies and computational experiments which are conducted to test the performance of the math-heuristic algorithm. We conclude with a few remarks and future research directions in Section 6 .

\section{Literature review}

Several approaches have been proposed in the literature to deal with unforeseen irregularities. These approaches can be categorized into two based on the timing of handling disruptions: (1) Preventative actions can be taken to address disruptions proactively; (2) Following a disruptive event, flight schedules, aircraft routings, crew pairings and passengers can be repaired reactively. Kohl et al. (2007) provide an overview of planning processes in the airline industry and described the general framework of disruption management. We refer to Clausen et al. (2010) for a review on disruption management.

Robust scheduling, which is a proactive way of handling disruptions, is widely studied in the literature. The objective is to generate schedules which are less sensitive to disruptions while minimizing the impact of propagated delays. Lan et al. (2006) present a model to minimize passenger misconnections by re-timing the departure times. Aloulou et al. (2013) and Ben Ahmed et al. (2017) adjust flight departure times to enhance the robustness of aircraft routings. Duran et al. (2015) propose a robust airline scheduling model considering the uncertainty of block-times. They use cruise time controllability introduced by Aktürk et al. (2014) together with idle time insertion to satisfy passenger connection service levels. Şafak et al. (2017) devise a two-stage algorithm that solves aircraft-path assignment and robust schedule design sequentially. Jamili (2017) propose a robust mathematical model for the integrated aircraft routing and scheduling problem by considering its impact on the fleet assignment problem. Marla et al. (2018) focus on constructing robust aircraft routes to minimize the disruptive effects of flight delays. On the other hand, Ben Ahmed et al. (2018) consider the robust integrated maintenance aircraft routing and crew pairing problem.

Proactive approaches might not be sufficient to absorb delays and recover all entities. Therefore, airlines take recovery actions reactively to reduce operational costs and to maintain passenger convenience following the occurrence of an unforeseen event. Since aircraft is the scarcest resource, many studies have been conducted about the aircraft recovery problem. Teodorovic and Guberinic (1984) first introduce the concept of aircraft unavailability and propose a model to minimize total delay with one less aircraft. Jarrah et al. (1993) formulate the aircraft recovery problem as a minimum cost network flow problem and use network flow algorithms to solve it. Rosenberger et al. (2003) model the aircraft recovery problem as a set-packing problem with an aim to minimize the cost of rerouting and cancellation. They reduce the problem size by developing an aircraft selection heuristic which determines the subset of aircraft to reroute. Abdelghany et al. (2008) propose a decision support tool for aiding flight controllers during irregular operations. They integrated a schedule simulation model and a resource assignment optimization model to be used within a rolling horizon scheme. The schedule simulation model detects future flight delays based on resource availability and legality rules whereas the optimization model is responsible for determining recovery actions. Khaled et al. (2018) propose a multi-criteria repair/recovery approach for the tail assignment problem. Many of these disruption management problems require rapid resolution. Vink et al. (2020) present a new approach that can be used as an operational decision support tool for aircraft recovery problem to obtain a solution in less than $1 \mathrm{~min}$.

Passengers are directly affected from re-timing and cancellation decisions made for flights. Xiong and Hansen (2013) discuss the connection between passenger-related parameters and airlines' flight cancellation decisions. Since passenger schedules highly depend on other resources, passenger recovery is integrated with aircraft and crew recovery problems in the literature. Bratu and Barnhart (2006) propose a passenger centric multi-commodity network flow model to find the optimal trade-off between passenger disruption and airline operating costs. Jafari and Zegordi (2010) integrate aircraft and passenger recovery decisions. They present an assignment model for recovering both aircraft and passengers simultaneously with the objective of minimizing the sum of aircraft assignment costs, delay costs, cancellation costs, and disrupted passenger costs. For integrated aircraft and passenger recovery problem, Bisaillon et al. (2011) propose a large neighborhood search algorithm which consists of three phases: construction, repair and improvement. In the first phase, a feasible rotation for each aircraft was constructed by changing aircraft type assignment, cancelling or delaying 
flights to some extent. In the repair phase, disrupted passenger itineraries were re-accommodated to alternative paths by repeatedly solving shortest path problems. In the last phase, it was allowed to delay flights for a certain amount of time in an attempt to accommodate additional passengers while also preserving the feasibility. Sinclair et al. (2014) extend this model by introducing a number of refinements in each phase to improve the solution quality. Petersen et al. (2012) study an integrated airline recovery problem using a single-day horizon, and propose a separate mixed-integer mathematical model for the schedule, aircraft, crew, and passenger recovery problems. They utilize a Benders decomposition scheme together with the column generation approach to achieve the coordination among these four mathematical models. They place a 30-min threshold of computation time for the overall problem; they also propose a sequential recovery algorithm to handle larger problems. Maher (2015) draws an analogy between knapsack and passenger recovery problems. In this context, aircraft with a fixed seat capacity corresponds to a knapsack whereas passengers represent the items to be put into the knapsack. A novel approach is presented at which cancellation variables are modeled as knapsack variables to demonstrate the possible reallocation options for passengers in the case of a cancellation. Overall aim is to find the optimal redistribution of passengers from cancelled legs to alternative operating flights. He applied column-and-row generation solution approaches to reduce the computation time. Zhang et al. (2016) propose a three-stage math-heuristic framework to solve joint aircraft scheduling and passenger itinerary recovery problem in sequential manner. Arikan et al. (2017) develop a new flight network based representation to capture interdependencies between aircraft, crew members and passengers. Kenan et al. (2018) integrate the flight scheduling, fleet assignment, and aircraft routing decisions, while considering different demand scenarios. In a different context, Montlaur and Delgado (2020) show that there is a trade-off between the operational efficiency of flights and fairness for passengers when assigning an on-ground tactical delay at the origin airport due to the reduced airport capacity at destination. This also highlights the importance of integrated recovery approaches.

In contrary to our study, most of the previously mentioned studies do not consider the possibility to use cruise speed control to prevent delays. Cook et al. (2009) introduce delay recovery opportunities which become available by speeding up flights to some extent. They focus on the trade-off between reducing fuel cost and environmental impact versus reducing delays. In order to quantify this, they define a cost index parameter which calculates the ratio of time-related costs to fuel-related costs dynamically. Dynamic estimation of cost terms was intended to aid airlines to control cruise speeds and manage flight delay costs. Aktürk et al. (2014) put an emphasis on flight time controllability and its environmental implications while considering the trade-off between delay minimization and fuel consumption. Although higher cruise speed allows recovery in a shorter time horizon, it leads to higher fuel burn and $\mathrm{CO}_{2}$ emission. Therefore they develop a nonlinear mixed integer recovery optimization model which takes the cruise speed as a decision variable, and adopted a conic quadratic optimization approach to mitigate computational difficulty. Marla et al. (2017) integrate disruption management with flight planning, which enables changes in the flight speed. Using a time-space network, they make multiple copies of flights representing different discrete departure times and cruise speeds. Gürkan et al. (2016) integrate robust schedule design, aircraft fleeting and routing problems while using cruise speed control.

In sum, airlines design their initial schedules relying on the assumption that flights will operate as planned and all resources will be available on time. However, on or before the day of operation, some disruptions might occur due to irregularities including adverse weather conditions, mechanical failures and maintenance delays. In order to lower the operational costs and maintain the passenger convenience, disruption management becomes a crucial field in the literature. Proactive and reactive models are developed to handle disruptions as discussed above. Earlier studies mostly address aircraft recovery problem since aircraft is the scarcest resource. For the other entities, crew members and passengers, sequential approaches are adopted. In the recent years, passenger convenience has attained more importance and it becomes crucial to capture dependencies within the system. Therefore, there is an emerging trend towards developing integrated recovery approaches. In this study, we work on airline rescheduling within the context of aircraft unavailability period. We address integrated aircraft and passenger recovery problem and contribute to the reactive disruption management literature by proposing a novel math-heuristic algorithm which makes individual passenger itinerary based recovery decisions under seat capacity limitations.

\section{The math-heuristic algorithm}

In this study, we work on integrated aircraft and passenger schedule recovery problem and deal with disruption scenarios which stem from the unavailability of aircraft. We use a schedule which is constructed with respect to hub-to-spoke paradigm and utilize flight block structure. A block can be defined as a set of flights which involves an initial outbound flight from hub to a demand point, its return flight to hub and all flights in between. Note that the aircraft which is scheduled to operate a particular block is at hub airport both at the beginning and at the end of each block. It is important in terms of keeping track of aircraft's location. We consider disrupted flights in block structure and evaluate each block as a whole. That is to say, any cancellation decision holds for the rest of the flights within the same block or all of them are assigned to the same type.

For given flight schedule, unavailable aircraft and disruption period, our objective is to come up with a recovery plan which maximizes the profit of airline while mitigating passenger dissatisfaction. We consider three recovery actions:

(1) Cruise time controllability: We do not take cruise times as fixed; instead, we provide flexibility to shorten cruise times to some extent by speeding up aircraft which are originally scheduled to operate at Maximum Range Cruise (MRC) speed. This enables AOCC to accommodate disrupted flight blocks into the rotation of an operating aircraft if using idle times and re-timing the departure times of the existing flights are not sufficient to open up a place for that block.

(2) Exchange of aircraft: We use the term exchange instead of swap. In the literature, swap corresponds to the change in rotations of two operating aircraft. Exchange is different in the sense that we do not consider a switch between two operating aircraft. We allow a switch only if one is operating and the other is unavailable. Therefore, flights which are initially assigned to the unavailable aircraft are flown by that operating aircraft. Instead, flights of that particular operating aircraft are cancelled as they are symbolically assigned to the unavailable one. We also allow aircraft of different types to be exchanged.

(3) Cancellation: During disruption periods, it might not be feasible for airlines to operate all flights due to limited resources, making cancellation inevitable. We cancel some flight legs to open up time in the schedule and to preserve feasibility. We provide flexibility over the cancellation set and enable AOCC to cancel less profitable flights. Here, ticket price is not the only measure for the profitability. We also consider passenger recovery options along with ticket price.

In a disruption scenario, we assume that we know the unavailable aircraft, duration of the disruption and affected flights which are initially assigned to that unavailable aircraft. Given this unavailability duration, we determine the disrupted flight blocks. We propose an integrated math-heuristic framework utilizing the aforementioned recovery tools. Due to the complexity of the problem, we handle the aircraft and passenger recovery with a two-phased formulation. In the first 


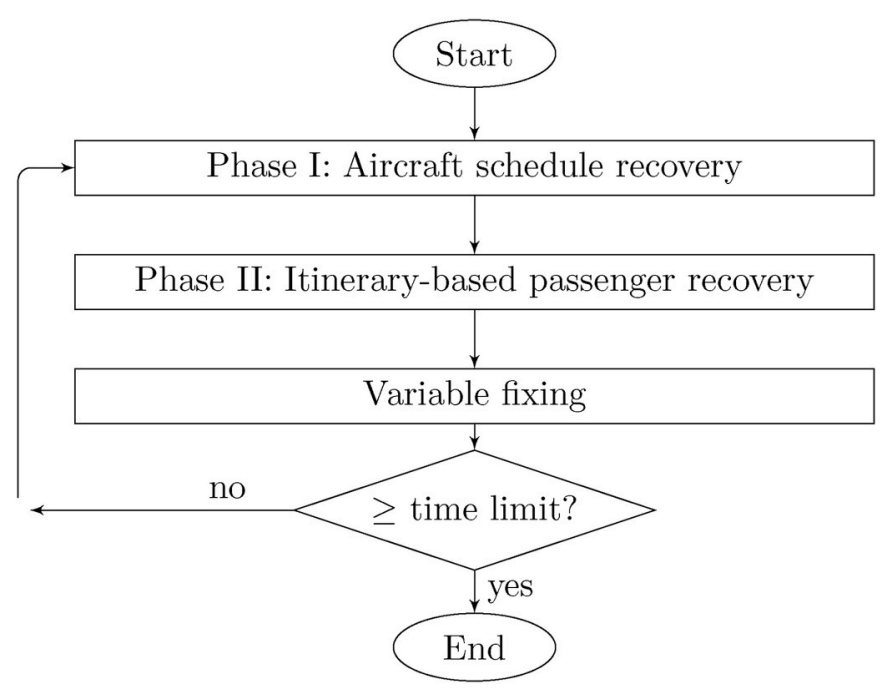

Fig. 1. Flow chart of the Math-heuristic Algorithm.

phase, we develop a mathematical model to solve aircraft recovery problem. Three decisions can be given for a disrupted flight block: (1) It can be accommodated into the existing schedule by adjusting cruise and departure times; (2) it can be flown by another aircraft after an exchange of unavailable aircraft and an operating one; or (3) it can be cancelled. As we work on integrated networks where passenger itineraries and aircraft routings are superimposed, we put a special emphasis on passengers in the second phase. We make individual passenger itinerary based recovery decisions and calculate the actual profit value. Depending on this calculation, we fix some assignment variables modeled in Phase I to control cancellation or operation of certain flights. Under a predetermined time limit, we solve this integrated problem in an iterative manner. Note that in Phase II, the result could depend on the order in which the heuristic visits each flight and canceled itinerary. Therefore, the solution obtained at the end cannot guarantee the optimality. The proposed math-heuristic algorithm is depicted in Fig. 1.

\subsection{Phase I: mathematical formulation for aircraft recovery}

In this section, we present a nonlinear mathematical model developed for aircraft recovery problem to be used at the first phase of our math-heuristic algorithm. We first list sets, parameters, decision variables used in model and then provide constraints along with the objective function.

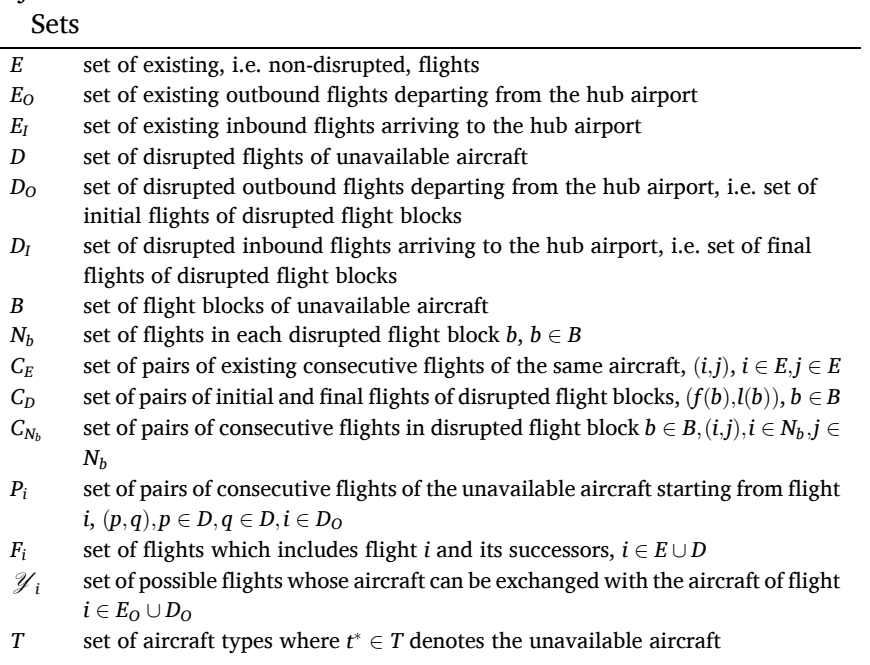

\section{Parameters}

$\begin{array}{ll}p(i) & \text { predecessor of flight } i \in E \\ f(b) & \text { first flight of block } b \in B \\ l(b) & \text { last flight of block } b \in B \\ t(i) & \text { aircraft type of flight } i \in E \\ l_{i}^{t} & \text { lower bound for the cruise time of flight } i \in E \cup D \text { with aircraft type } t \in T \\ u_{i}^{t} & \text { upper bound for the cruise time of flight } i \in E \cup D \text { with aircraft type } t \in T \\ d e p_{i}^{l} & \text { lower bound for the departure of flight } i \in E \cup D \\ d e p_{i}^{u} & \text { upper bound for the departure of flight } i \in E \cup D \\ \eta_{i} & \text { non-cruise time of flight } i \in E \cup D \\ \tau_{i}^{t} & \text { turnaround time required to prepare aircraft type } t \in T \text { for the next flight after } \\ & i \in E \cup D \\ \kappa^{t} & \text { seat capacity of aircraft type } t \in T \\ \mu_{i} & \text { number of passengers of flight } i \in E \cup D \\ \pi_{i} & \text { ticket price of flight } i \in E \cup D \\ \sigma_{i} & \text { spill cost per passenger of flight } i \in E \cup D \\ \varphi_{i} & \text { overnight cost per passenger of flight } i \in E \cup D\end{array}$

\section{Decision variables}

$\begin{array}{ll}\text { dep }_{i} & \text { departure time of flight } i \in E \cup D \\ \operatorname{arr}_{i} & \text { arrival time of flight } i \in E \cup D \\ f_{i}^{t} & \text { cruise time of flight } i \in E \cup D \text { with aircraft type } t \in T \\ x_{i j} & \begin{array}{l}\text { if flight } i \in E_{I} \cup D_{I} \text { is followed by flight } j \in E_{O} \cup D_{O} \text { in the route of an aircraft, } \\ \text { and } 0 \text { otherwise }\end{array} \\ y_{i j} & \text { if aircraft of flight } i \in E_{O} \cup D_{O} \text { and } j \in E_{O} \cup D_{O} \text { are exchanged, and } 0 \text { otherwise } \\ z_{i}^{t} & \text { if aircraft type } t \text { is assigned to flight } i, i \in E \cup D, t \in T, \text { and } 0 \text { otherwise }\end{array}$

\subsubsection{Model}

$\max \sum_{t \in T \backslash t^{t} i \in E \cup D} \sum_{i} \min \left\{\mu_{i}, \kappa^{t}\right\} z_{i}^{t}-\sum_{t \in T \backslash t^{*} i \in E \cup D} \sum_{i}\left(f_{i}^{t}\right)-\sum_{i \in E \cup D} \varphi_{i} \min \left\{\mu_{i}, \kappa^{t^{t}}\right\} z_{i}^{t^{t}}$
$-\sum_{i \in E_{O} j \in D_{O}} \sum_{k \in F_{j}} \sigma_{k} \max \left\{0, \mu_{k}-\kappa^{t(i)}\right\} y_{i j}$

s.t. $\sum_{t \in T} z_{i}^{t}=1 \quad i \in E \cup D$

$z_{i}^{t}=z_{j}^{t} \quad i \in D_{O}, j \in N_{b}: f(b)=i$

$\sum_{i \in E_{I}} x_{i n}+\sum_{i \in E_{O} \cup D_{O}} x_{m i}+\sum_{i \in E_{O}} y_{i n}+z_{n}^{t^{*}} \geq 1 \quad(n, m) \in C_{D}$

$\left|z_{j}^{t}-z_{i}^{t}\right| \leq\left(1-x_{i j}\right) \quad i \in E_{I}, j \in D_{O}, t \in T$

$\left|z_{j}^{t}-z_{i}^{t}\right| \leq\left(1-x_{j i}\right) \quad i \in E_{O}, j \in D_{I}, t \in T$

$\sum_{i \in E_{I}} x_{i j} \leq 1 \quad j \in D_{O}$

$\sum_{i \in E_{O}} x_{j i} \leq 1 \quad j \in D_{I}$

$\sum_{j \in D_{O}} x_{i j} \leq 1 \quad i \in E_{I}$

$\sum_{j \in D_{I}} x_{j i} \leq 1 \quad i \in E_{O}$

If $x_{i j}=1$, then $\operatorname{arr}_{i}+\sum_{t \in T} \tau_{i}^{t} z_{i}^{t} \leq d e p_{j} \quad i \in E_{I}, j \in D_{O}$

If $x_{j i}=1$, then $a r r_{j}+\sum_{t \in T} \tau_{j}^{t} z_{j}^{t} \leq d e p_{i} \quad i \in E_{O} \cup D_{O}, j \in D_{I}$

$\sum_{i \in E_{O}} \sum_{j \in E_{O}} y_{i j}=0$ 
$\sum_{i \in E_{O} j \in D_{O}} y_{i j} \leq 1$

$y_{i j}=y_{j i} \quad i \in E_{O} \cup D_{O}, j \in \mathscr{Y}_{i}$

$\left|z_{p(i)}^{t}-z_{i}^{t}\right| \leq \sum_{j \in \mathscr{Y}_{i}} y_{i j} \quad i \in E_{O}, t \in T$

$\left|x_{i n}-x_{m j}\right| \leq \sum_{k \in \mathscr{Y}_{j}} y_{j k} \quad(i, j) \in C_{E},(n, m) \in C_{D}$

If $\sum_{n \in D_{O}} x_{i n}+\sum_{k \in \mathscr{Y}_{j}} y_{j k}=0$, then $\quad \operatorname{arr}_{i}+\sum_{t \in T} \tau_{i}^{t} z_{i}^{t} \leq d e p_{j} \quad(i, j) \in C_{E}$

$y_{i j} \leq z_{k}^{t^{*}} \quad i \in E_{O}, j \in D_{O}, k \in F_{i}$

$y_{i j} \leq z_{k}^{t(p(i))} \quad i \in E_{O}, j \in D_{O}, k \in F_{j}$

If $y_{i j}=1$, then $\operatorname{arr}_{p}+\tau_{p}^{t(p(i))} \leq \operatorname{dep}_{q} \quad i \in E_{O}, j \in D_{O},(p, q) \in P_{j} \cup(p(i), j)$

$\left|x_{i n}-x_{m k}\right| \leq 1-y_{j k} \quad k \in \mathscr{Y}_{j},(i, j) \in C_{E},(n, m) \in C_{D}$

$\operatorname{dep}_{i}+\sum_{t \in T} f_{i}^{t}+\eta_{i}=\operatorname{arr}_{i} \quad i \in E \cup D$

If $z_{i}^{t^{*}}=0$, then $d e p_{i}^{l} \leq d e p_{i} \leq d e p_{i}^{u} \quad i \in E \cup D$

$l_{i}^{t} z_{i}^{t} \leq f_{i}^{t} \leq u_{i}^{t} z_{i}^{t} \quad i \in E \cup D, t \in T \backslash t^{*}$

If $z_{f(b)}^{t}=1$, then $\operatorname{arr}_{i}+\tau_{i}^{t} \leq d e p_{j} \quad(i, j) \in C_{N_{b}}, b \in B, t \in T \backslash t^{*}$

$x_{i j} \in\{0,1\} \quad i \in E_{I} \cup D_{I}, j \in E_{O} \cup D_{O}$

$y_{i j} \in\{0,1\} \quad i \in E_{O} \cup D_{O}, j \in E_{O} \cup D_{O}$

$z_{i}^{t} \in\{0,1\} \quad i \in E \cup D, t \in T$

Our objective is to maximize the profit of airline in the case of a disruption. Profit value can be calculated as follows: Revenue - Fuel and $\mathrm{CO}_{2}$ Emission Cost - Overnight Passenger Cost - Spilled Passenger Cost. First component of the objective is the revenue generated by ticket sales of operating flights and it is directly proportional to the number of passengers assigned to them. Note that cancelled flights do not contribute to the revenue. In our setting, there are three types of operating flights: (1) flights which are accommodated into the rotation of an available aircraft thanks to the cruise time controllability, (2) flights which are initially assigned to the unavailable aircraft but recovered after an exchange of aircraft, and (3) flights which are not subject to change and operate as planned. It is important to mention that in order to operate flights by shortening cruise times, additional fuel and emission cost is incurred. Likewise, due to the insufficient seat capacity, some passengers might be spilled after an exchange of aircraft. They are also penalized in the objective function.

Second term reflects the nonlinear fuel and $\mathrm{CO}_{2}$ emission cost which corresponds to the largest portion of the operating expenses. In order to calculate the fuel burn, we use the cruise stage fuel flow model proposed by the Base of Aircraft Data (BADA) project of EUROCONTROL (2012) as in Şafak et al. (2017). We express this cost component as a function of cruise time (f) and aircraft type ( $t$ ) for each flight. It can be calculated by using the following formula:

$c_{i}^{t}\left(f_{i}^{t}\right)=\left\{\begin{array}{cc}c_{0}\left(\alpha_{i}^{t} \frac{1}{f_{i}^{t}}+\beta_{i}^{t} \frac{1}{\left(f_{i}^{t}\right)^{2}}+\gamma_{i}^{t}\left(f_{i}^{t}\right)^{3}+\nu_{i}^{t}\left(f_{i}^{t}\right)^{2}\right), & \text { if } z_{i}^{t}=1, t \neq t^{*} \\ 0, & \text { if } z_{i}^{t}=0,\end{array}\right.$ where $\alpha, \beta, \gamma, \nu$ are the non-negative aircraft-specific fuel consumption coefficients and $c_{0}$ denotes the unit fuel and emission cost $(\$ \backslash \mathrm{kg}$ ). This formula indicates that the nonlinear fuel and $\mathrm{CO}_{2}$ emission cost is incurred only if a flight is assigned to an operating aircraft. We propose strong mixed-integer conic quadratic (MICQ) formulations to overcome the computational difficulties of nonlinear fuel burn and emission functions. As the cost function is defined based on the indicator assignment variable, it is discontinuous making the epigraph $E_{F}=\left\{(f, t) \in R^{2}: c(f) \leq t\right\}$ nonconvex. Şafak et al. (2017) represent the convex hull of $E_{F}$ by conic quadratic inequalities as follows:

$t \geq c_{0}(\alpha p+\beta q+\gamma r+v h)$

$z^{2} \leq p f$

$z^{4} \leq f^{2} q z$

$f^{4} \leq z^{2} r f$

$f^{2} \leq h z$

Moreover, each inequality can be represented by conic quadratic inequalities. Then, we become able to solve the model proposed in Phase I using IBM ILOG CPLEX Optimization Studio 12.6.2. We solve the reduced problem at Phase I to optimality without any gap. A more detailed information regarding the convexification of the epigraph and representation by conic quadratic inequalities can be found in Aktürk et al. (2009).

Third term is a penalty for overnight passengers who have to stay overnight at their origin locations due to the cancellation of flights. Finally, the last term represents the cost of spilled passengers due to inadequate seat capacity of exchanged aircraft.

Constraint (1) ensures that each flight is assigned to exactly one aircraft type. Note that set of aircraft types encompasses the unavailable aircraft as well, $t^{*} \in T$. If flight $i$ is assigned to the unavailable aircraft $t^{*}$, i.e. $z_{i}^{t^{*}}=1$, then this indicates that flight $i$ is cancelled. Constraint (2) maintains the block structure that any decision given holds for the rest of the flights within the same block. That is, they are assigned to same aircraft type. Constraint (3) guarantees that a disrupted flight block is either recovered or cancelled. It can be placed into the schedule of an operating aircraft, flown by another aircraft through exchange or cancelled. When a disrupted flight block is accommodated into the existing schedule of an operating aircraft, it comes before or after an operating flight making $x_{i j}=1$ or $x_{j i}=1$. In such cases, constraints (4)(5) assign the aircraft type of that particular aircraft to initially disrupted flight block. Constraints (6)-(7) ensure that a disrupted flight follows or is followed by at most one existing flight. Similarly, constraints (8)-(9) assure that an existing flight immediately precede or is preceded by at most one disrupted flight. For the flights which are assigned to the same aircraft, we need to define order of succession among operations. Constraint (10) guarantees that if a disrupted outbound flight follows an inbound existing flight, then the later should depart after the arrival time of flight $i$ plus its turnaround time. Same limitation holds if a disrupted inbound flight is followed by an existing outbound flight as in constraint (11).

Since we want to keep the new schedule as similar as possible to the existing one, we only let exchange of aircraft at hub airport on condition that one is operating and the other is unavailable. Constraint (12) indicates that exchange of two operating aircraft is not an allowable option whereas constraint (13) puts a limit on the number of exchanges. Symmetry of the exchange decision is maintained by constraint (14). Constraint (15) makes a connection between aircraft type assignment and exchange decisions. If there is no exchange between the aircraft of flight $i$ and flight $j$, aircraft of flight $i$ has the same fleet type assignment with its predecessor $p(i)$ as planned. This enables to preserve the initial schedule. Again, if there is no exchange before the departure of flight $j$ and the disrupted flight block which is represented by $(n, m)$ is operated between existing consecutive flights $i$ and $j$, then constraint (16) guarantees that $x_{i n}=x_{m j}$, keeping the sequence of existing flights as in the original schedule. Constraint (17) assures that if there is no disrupted 
flight succeeding flight $i$ and aircraft assignment does not change after it, then the requirement for the minimum aircraft connection time needs to be satisfied for existing flight pair $(i, j)$. On the other hand, if aircraft of flight $i$ and unavailable aircraft are exchanged at hub airport, then constraint (18) assigns flight $i$ and its successors to unavailable aircraft which means that they are all cancelled. Besides, flight $j$ and previously disrupted flights which follow flight $j$ are recovered. Constraint (19) assigns these flights to the aircraft type of $p(i)$. As the initially disrupted flight pairs are assigned to an operating aircraft, constraint (20) controls the precedence relations between consecutive pairs including $(p(i), j)$. Constraint (21) enforces that $x_{i n}=x_{m k}$ for disrupted flight pair $(n, m)$ if the aircraft of flight $j$ and $k$ are exchanged, i.e. $y_{j k}=1$.

Constraint (22) defines the arrival time of flight $i$ by taking both cruise time and non-cruise time into consideration. Constraints (23)(25) are valid for the flights which are not cancelled. Although we allow re-timing departure times of each flight, we need to bound these changes within allowable ranges for each flight due to marketing requirements. Constraint (23) applies lower and upper bounds for departure of each flight which is assigned to an operating aircraft. Constraint (24) makes sure that cruise time falls into a range which is determined with respect to the aircraft type and allowable compression rate due to technical limitations. Constraint (25) controls the precedence relationship within an operating flight block. Constraints (26)-(28) define the domain of each decision variable.

\subsection{Phase II: itinerary-based passenger recovery algorithm}

Each passenger in the system can be characterized by the itinerary that $\mathrm{s}$ /he follows. An itinerary might either be a single flight leg or a composition of multiple legs. Therefore, passengers who travel on the same flight do not necessarily belong to the same itinerary. In the first phase, we do not make a distinction between passengers who are on the same flight and accept them equal regardless of their itineraries. In this regard, we do not differentiate between direct and connecting passengers as well. For instance, if a flight is canceled as a solution to the optimization problem in Phase I, then the objective value is reduced by the revenue lost (ticket price of that leg times the demand) of that particular leg, without considering the effect of that cancellation in successive flights in terms of the profit and seat capacity. Moreover, passenger re-accommodation is not considered in Phase I, even if there exist alternative paths between certain origin and destination pairs with available seat capacity. Therefore, the objective value obtained at the end of Phase I only gives us an approximate profit of the airline.

In this section, we propose Passenger Recovery Algorithm (PRA) which makes individual itinerary based recovery decisions. The algorithm starts with the objective value of Phase I and calculate the actual profit by making adjustments on it in a dynamic manner. Recall that in the previous phase, passengers of cancelled flights do not contribute to the revenue. With PRA, if a passenger is recovered then the ticket price which s/he pays originally is added to the profit value. Also, this passenger may not have to stay overnight anymore, so the objective is adjusted by adding previously subtracted overnight cost.

For passenger re-accommodation, we consider two options in PRA as long as the seat capacity is not exceeded: (1) A passenger can be reassigned to an alternative direct flight which shares the same origin and destination airports with the passenger's original itinerary, or (2) the passenger can be re-routed and reach to the destination over another hub airport, $H$. Here, any re-accommodation decision given for a particular passenger might directly affect the others. This is because, if a previously available seat is occupied, then it is no longer be a reaccommodation option for other disrupted passengers. To that end, we prioritize the affected passengers with respect to their contributions to the objective value in PRA. Consequently, we could calculate the actual cancellation cost by evaluating each passenger itinerary while considering the seat capacity limitations as opposed to multiplying the number of passengers in a cancelled flight with a fixed penalty coefficient to approximate the cancellation cost as commonly done in the literature. Here, it is important to mention that passengers can buy itinerary tickets that can compromise more than one leg and there might exist several tariffs for the same itinerary. In this study, we generate recovery options for the passengers with respect to the disrupted flight legs at which they are assigned to. For instance, lines 9-32 in PRA correspond to the recovery options for the connecting passengers whose $1^{\text {st }}$ leg of connection is disrupted and so on. Therefore, we need to estimate the ticket price for each flight leg instead of an itinerary. This allows us to approximate the cost of cancellation in the case of a disruption.

Given a cancelled flight, we first determine the set of passenger itineraries which are disrupted due to the cancellation of that flight. Then, among those itineraries, we give the highest priority to the ones which represent connecting passengers whose first leg of connection is disrupted. The reason is that, otherwise, they would also miss their continuing flights and this may lower the objective value substantially. Such type of passengers are followed by the ones whose second leg of connection is disrupted and lastly direct passengers have the least priority. The algorithm proceeds with respect to this prioritization. We assume that passenger itineraries are composed of either a single flight leg or two legs. The logic followed in the algorithm can be extended for multiple legs as well. Cases 1-3 are associated with the connecting passengers whose first leg is cancelled. Cases 4-7 show the steps when the second leg of a connection is disrupted. Lastly, rest of the cases are related to direct passengers. We use the following additional notation for PRA and the algorithm can be found in Appendix A:

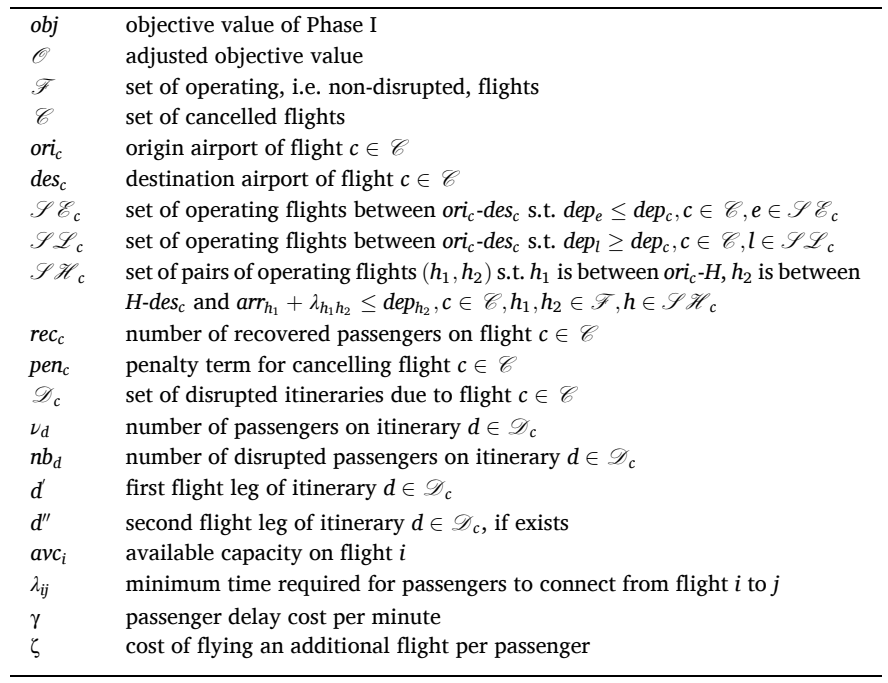

Let $c \in \mathscr{C}$ be a cancelled flight which is supposed to depart from ori $i_{c}$ and arrive at $\operatorname{des}_{c}$. Also, let $n b_{d}$ denote the number of passengers on itinerary $d$ who are disrupted due to the cancellation of flight $c$.

Case 1, 5 and 8: Let $r \in \mathscr{S} \mathscr{L}_{c}$ be a direct flight between ori $_{c}$ and $\operatorname{des}_{c}$ with a later departure time. Therefore, it can be interpreted as a late alternative for the disrupted flight $c$. In order not to violate seat capacity restrictions, $\min \left\{n b_{d}, a v c_{r}\right\}$ passengers can be recovered without adding extra flights to their paths. However, passengers on $r$ arrive at $d_{e} s_{c}$ late by $\Delta t$ minutes. In Case 5 and $8, \min \left\{n b_{d}, a v c_{r}\right\} \gamma \Delta t$ stands for the inconvenience cost incurred due to passenger delay. In Case 1, those recovered passengers can continue their connections with flight $d^{\prime \prime}$ as the minimum connection time is not violated. Since they arrive at their final airport, des $_{d^{\prime \prime}}$. as planned, no delay cost is incurred.

Case 2, 6 and 9: For the passengers who cannot be recovered by either earlier or later direct flights, an alternative path can be taken into consideration. They can reach to their destination airports over another hub airport, $H$. In this regard, let $r \in \mathscr{S}_{\mathscr{H}_{c}}$ where and $r_{2}$ denote the two operating flight legs between ori $i_{c}-H$ and $H-\operatorname{des}_{c}$ respectively. By adding an extra flight, $\min \left\{n b_{d}, a v c_{r_{1}}, a v c_{r_{2}}\right\}$ passengers can be rerouted 
and they can follow ori $_{c}-H-\operatorname{des}_{c}$ path. Taking the minimum of $a v c_{r_{1}}$ and $a v c_{r_{2}}$ provides completeness and prevents passengers to stay at hub airport $H$. Due to this additional flight, people need to deal with extra check-in procedures. It also increases the risk of missing the upcoming flights. Therefore, besides the passenger delay cost, adding an extra flight is penalized as well. The term $\min \left\{n b_{d}, a v c_{r_{1}}, a v c_{r_{2}}\right\} \zeta$ can be interpreted as a disincentive for adding an extra flight to an itinerary.

Note that this recovery option is feasible only within connected networks. In such networks, spoke airports are served from different hubs and hub airports are connected by regular flights. Unless there are alternative ways to reach a particular airport, rerouting cannot be possible. In Case 2, delay on the first leg of connection which occurs due to this additional flight has no impact on the passengers' arrival time to destination airport. Hence, no delay cost is incurred. However, in Cases 6 and 9 , rerouted passengers are delayed by $\Delta t$ minutes which is also considered in the actual profit calculation.

Case 3, 7 and 10: As a terminating condition, if the passengers of itinerary $d$ cannot be recovered by any means, then they have to stay overnight at origin airport and the penalty term pen $_{c}$ is updated accordingly. pen $_{c}$ is intended to measure the loss of profit if the airline prefers to cancel flight $c$ considering each passenger itinerary separately. Observe that in Case $3 n b_{d} \pi_{d^{\prime \prime}}$ is subtracted from the objective value different from the other cases. To understand the logic behind this adjustment, let us consider a connecting passenger whose first leg of connection, flight $c$, is cancelled. A passenger who is supposed to be at $\operatorname{des}_{c}$ has to stay at origin airport, ori $i_{c}$ as a result of this cancellation. However, the continuing flight of that passenger departs from $d_{e}$. Although second flight leg is operated as planned, the passenger cannot take place on this flight. Therefore, revenue can not be generated from the ticket sales of the second leg for such passengers.

Case 4: Let $e \in \mathscr{S} \mathscr{E}_{c}$. That is, it shares the same origin and destination airports with flight $c$ with an earlier departure time. So, it can be evaluated as an early alternative for the second flight leg of the original itinerary. Since the passenger connectivity is still satisfied between first and second leg, $\min \left\{n b_{d}, a v c_{e}\right\}$ passengers can be recovered by allocating available seats. Note that those passengers can be informed in advance so that they will not be entitled to any compensation. As they reach the destination airport earlier, no delay cost is incurred.

\subsection{Integrated search algorithm with variable fixing}

In order to maintain the integration between two phases, we propose the Integrated Search Algorithm (ISA) which is developed based on the variable fixing idea. The objective is to find a better solution in terms of the profit by enforcing some flights to operate or to cancel. For ISA, we first define two new sets which are denoted as $\mathscr{F} \mathscr{O}$ and $\mathscr{F} \mathscr{C}$ to cover fixed operating and cancelled flights. The algorithm starts with solving Phases I and II and takes the cancellation set as input. For each cancelled flight, it checks whether it is possible to recover all disrupted passengers or not. Note that passengers who are assigned to the same flight can be recovered by different ways, so it is not necessary to make a single decision. If all recovery is possible for the cancelled flight $f^{*}$, i.e. $\exists f^{*} \in$ $\mathscr{C}_{k}$ st. $\quad \operatorname{rec}_{f^{*}}=\mu_{f^{*}}$, then it is added to fixed cancellation set. $\mathscr{F} \mathscr{C}$ is updated as follows: $\mathscr{F} \mathscr{C}=\mathscr{F} \mathscr{C} \cup\left\{f^{*}\right\}$. This is because, even with one less flight all passengers can be transported to their destination airports.

While updating $\mathscr{T} \mathscr{O}$, set of fixed operating flights, the following logic is used: Among all disrupted flights, the algorithm chooses the one which yields the highest penalty value to the airline, i.e. $c^{*}=$ $\operatorname{argmax}_{i \in \mathscr{C}_{k}}$ pen $_{i}$. Then $c^{*}$ is fixed to be operated: $\mathscr{F} \mathscr{O}=\mathscr{F} \mathscr{O} \cup\left\{c^{*}\right\}$. That is, for the next iteration $k$, the algorithm will make a search over the remaining flights while determining the cancellation set, $\mathscr{C}_{k}$. As $c^{*}$ is not a candidate for cancellation anymore, the solution space will be different. Fixing a binary assignment variable eliminates a branch in the solution space reducing the size of it. Unless the time limit is exceeded, the algorithm switches back to Phase I and the aircraft recovery model is solved with the following additional assignment constraints: $\sum_{t \in T \backslash\left\{t^{*}\right\}} z_{i}^{t}=1 \quad \forall i \in \mathscr{T} \mathscr{O}$ and $z_{i}^{t^{*}}=1 \quad \forall i \in \mathscr{F} \mathscr{C}$. Note that $\mathscr{C}_{k}$ differs at each iteration since the corresponding assignment variables are fixed. At each iteration, algorithm checks whether the current set of cancellation gives a better objective value or not. If so, the incumbent solution is updated but otherwise it is discarded. In sum, ISA works in an iterative manner and returns the best possible cancellation set as well as the profit value at the end.

Different from the notation in PRA, we add an index to $\mathscr{C}$ to represent the set of cancellation at each iteration $k$. The following additional notations are used for ISA and the algorithm can be found in Appendix B:

\begin{tabular}{ll}
\hline $\mathscr{T} \mathscr{O}$ & set of fixed operating flights \\
$\mathscr{F} \mathscr{C}$ & set of fixed cancelled flights \\
$\mathscr{C}_{k}$ & set of cancelled flights at iteration $k$ \\
$\mathscr{C}^{*}$ & cancellation set of the best possible solution \\
$o b j^{*}$ & objective value of the best possible solution \\
$t_{\text {total }}$ & total solution time \\
$\bar{t}$ & time limit \\
\hline
\end{tabular}

\section{Numerical example}

In this section, we give a numerical example to illustrate how recovery or cancellation decisions are made for flights of an unavailable aircraft. We take original flight schedule, corresponding passenger itineraries, characteristics of aircraft and disruption information as input. We consider a sample schedule for five aircraft, each completing its route at hub airport, ORD. Table 1 shows aircraft assignment, flight number, origin and destination airports, base ticket price, planned departure and arrival in local ORD time, planned flight time and the number of passengers for each flight in the sample schedule.

Fig. 2 (a) gives the time-space network representation of the original schedule. Horizontal and vertical axes represent time line and airports, respectively. Arcs which emanate from the origin airport at planned departure time and end at the destination airport after planned block time correspond to flight arcs. Ground arcs represent the turnaround time spent on ground which is required to prepare the aircraft for the next flight.

In this example, we assume that aircraft N03442-N03449, N15425N15438 and N27261 are Boeing 727-228, Boeing 737-500 and McDonnell Douglas 83 respectively. The number of seats of the aircraft are 134, 122 and 148. In the original schedule, for each flight we assume that aircraft fly at the most fuel efficient Maximum Range Cruise (MRC) speed and non-cruise stages take $30 \mathrm{~min}$. This implies that cruise stages last $30 \mathrm{~min}$ less than the planned flight times given in Table 1.

We assume that passenger itineraries, which are displayed in Table 2, are composed of either a single flight leg or two legs. Each flight is included as a single-flight itinerary. Moreover, itineraries which require connection of passengers are expressed with sequential flight numbers. Such passengers might require to switch aircraft and hence need some time to make a connection to a continuing flight. We set all minimum connection times to $30 \mathrm{~min}$.

In daily operations, some aircraft might become unavailable and hence flights cannot be operated as planned. On the given schedule, let us assume that aircraft N27261 undergoes an emergency maintenance at ORD airport and it is unavailable between 09:00-19:00. Apparently, flights 15, 16 and 17 are disrupted since the corresponding aircraft cannot depart at planned departure time. Although the departure of flight 18 does not coincide with the unavailability period, it is also affected. This is because the aircraft N27261 which supposed to be at ROC airport by 19:20 is still at ORD and hence it is not possible to operate ROC-ORD flight. With this unavailability information, two flight blocks (ORD-MSP-ORD and ORD-ROC-ORD) are disrupted.

One feasible solution is to cancel all of the flights which are initially assigned to the unavailable aircraft without making any further changes in the existing schedule. Characteristics of that solution approach is provided in Table 3. An alternative way of handling disruptions is to 
Table 1

Original schedule.

\begin{tabular}{|c|c|c|c|c|c|c|c|c|}
\hline $\begin{array}{l}\text { Tail } \\
\#\end{array}$ & $\begin{array}{l}\text { Flight } \\
\#\end{array}$ & $\begin{array}{l}\text { Dep. } \\
\text { Airport }\end{array}$ & $\begin{array}{l}\text { Arr. } \\
\text { Airport }\end{array}$ & $\begin{array}{l}\text { Dep. } \\
\text { Time }\end{array}$ & $\begin{array}{l}\text { Flight } \\
\text { Time }\end{array}$ & $\begin{array}{l}\text { Arr. } \\
\text { Time }\end{array}$ & \# of Pass. & $\begin{array}{l}\text { Base } \\
\text { Price }\end{array}$ \\
\hline \multirow[t]{3}{*}{ N03442 } & 0 & DEN & ORD & $07: 40$ & $02: 20$ & $10: 00$ & 110 & 168 \\
\hline & 1 & ORD & DEN & $14: 15$ & $02: 40$ & $16: 55$ & 130 & 168 \\
\hline & 2 & DEN & ORD & $19: 30$ & $02: 45$ & $22: 15$ & 117 & 168 \\
\hline \multirow[t]{4}{*}{ N15425 } & 3 & ORD & ATL & 08:15 & 02:15 & $10: 30$ & 119 & 165 \\
\hline & 4 & ATL & ORD & $12: 00$ & 02:15 & $14: 15$ & 118 & 165 \\
\hline & 5 & ORD & MCO & $16: 30$ & 03:00 & $19: 30$ & 104 & 150 \\
\hline & 6 & MCO & ORD & $20: 45$ & $02: 45$ & $23: 30$ & 94 & 150 \\
\hline \multirow[t]{4}{*}{ N15438 } & 7 & ORD & DEN & $07: 10$ & $02: 20$ & 09:30 & 121 & 168 \\
\hline & 8 & DEN & ORD & $11: 30$ & $02: 30$ & $14: 00$ & 119 & 168 \\
\hline & 9 & ORD & $\mathrm{IAH}$ & $15: 00$ & 03:00 & $18: 00$ & 110 & 187 \\
\hline & 10 & IAH & ORD & $20: 00$ & 03:00 & $23: 00$ & 99 & 187 \\
\hline \multirow[t]{4}{*}{ N03449 } & 11 & DFW & ORD & 09:50 & $02: 40$ & $12: 30$ & 126 & 187 \\
\hline & 12 & ORD & DFW & $15: 15$ & $02: 30$ & $17: 45$ & 127 & 187 \\
\hline & 13 & DFW & $\mathrm{IAH}$ & $18: 30$ & 01:15 & $19: 45$ & 97 & 199 \\
\hline & 14 & IAH & ORD & $20: 45$ & $02: 50$ & $23: 35$ & 110 & 187 \\
\hline \multirow[t]{4}{*}{ N27261 } & 15 & ORD & MSP & 09:30 & 01:55 & $11: 25$ & 108 & 128 \\
\hline & 16 & MSP & ORD & $12: 00$ & 01:50 & $13: 50$ & 143 & 128 \\
\hline & 17 & ORD & ROC & $17: 00$ & $02: 20$ & $19: 20$ & 123 & 227 \\
\hline & 18 & ROC & ORD & $20: 30$ & $02: 30$ & $23: 00$ & 109 & 227 \\
\hline
\end{tabular}

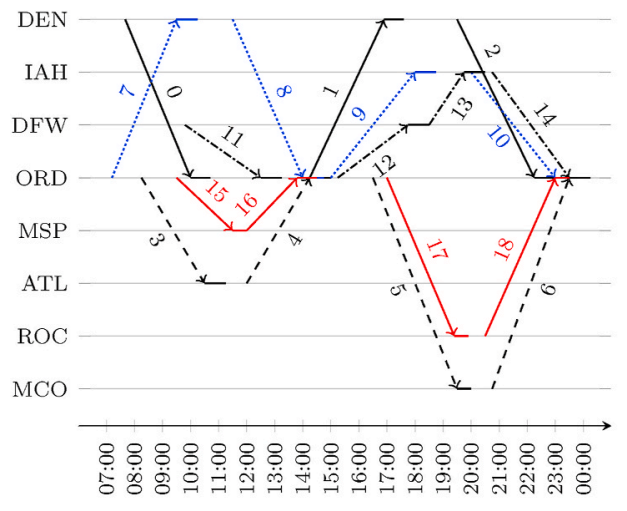

(a) Original schedule

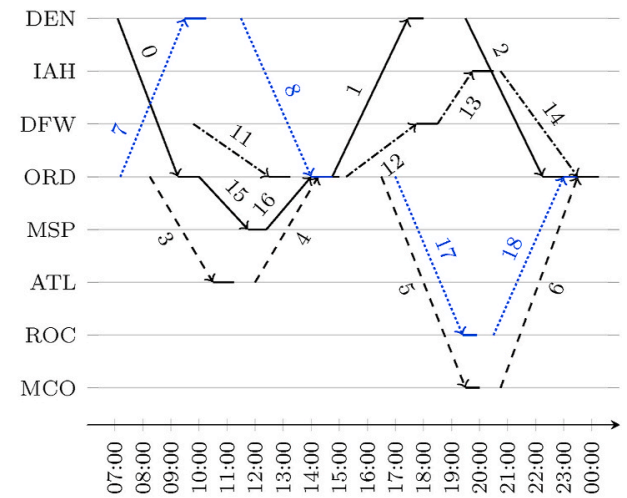

(b) Proposed schedule

Fig. 2. Time space network of schedules.

Table 2

Planned passenger itineraries.

\begin{tabular}{llll}
\hline Itinerary & \# of Pass. & Itinerary & \# of Pass. \\
\hline 0 & 73 & 10 & 99 \\
$0-5$ & 21 & 11 & 105 \\
$0-17$ & 16 & $11-17$ & 21 \\
1 & 130 & 12 & 109 \\
2 & 117 & 13 & 97 \\
3 & 119 & 14 & 110 \\
4 & 104 & 15 & 108 \\
$4-17$ & 14 & 16 & 94 \\
5 & 44 & $16-5$ & 18 \\
6 & 94 & $16-9$ & 10 \\
7 & 121 & $16-12$ & 18 \\
8 & 89 & $16-17$ & 13 \\
$8-5$ & 21 & 17 & 50 \\
$8-17$ & 9 & 18 & 109 \\
9 & 100 & & \\
\hline
\end{tabular}

consider recovery options along with cancellation. We utilize cruise time controllability as a recovery tool and allow the compression of cruise times up to $15 \%$. Moreover, we allow exchange of aircraft at hub airport (ORD) only if one is operating and the other is unavailable to preserve the initial schedule. We run our math-heuristic algorithm for this disruption scenario under the time limit of $60 \mathrm{~s}$. For the computation of cost components, we set the overnight cost per passenger to $\$ 50$, delay
Table 3

Comparison of results after each iteration.

\begin{tabular}{llll}
\hline & $\begin{array}{l}\text { All Cancellation } \\
\text { (Base Policy) }\end{array}$ & Iteration 1 & $\begin{array}{l}\text { Iteration 2 } \\
\text { (Proposed) }\end{array}$ \\
\hline Cancelled flights & $15,16,17,18$ & 5,6 & 9,10 \\
\# of recovered passengers & 0 & 242 & 273 \\
$\rightarrow$ Phase I & 0 & 242 & 242 \\
$\rightarrow$ Phase II & 0 & 0 & 31 \\
\# of overnight passengers & 483 & 218 & 198 \\
\% Imp. Overnight & - & 55.1 & 59.2 \\
\hline Total compression (min) & 0 & 34 & 34 \\
Total passenger delay (min) & 0 & 0 & 1815 \\
Compression cost (\$) & 0 & 471 & 471 \\
Passenger delay cost (\$) & 0 & 0 & 1815 \\
Overnight cost (\$) & 24150 & 11835 & 10834 \\
Objective (\$) & 90958 & 141344 & 142181 \\
\% Imp. Objective & - & 64.8 & 66.1 \\
\hline
\end{tabular}

cost per minute per passenger to $\$ 1.09$, unit cost of fuel to $1.2 \$ / \mathrm{kg}$ and unit cost of $\mathrm{CO}_{2}$ emission to $0.02 \$ / \mathrm{kg}$.

At iteration 1, first disrupted flight block which involves flights 15 and 16 is accommodated into the schedule of the aircraft N03442 between flights 0 and 1 . So as to open up sufficient time for this accommodation, speed of aircraft is adjusted. Cruise times of flights 0,15 and 16 are compressed by 14,10 and 10 min respectively, increasing the 
utilization of the aircraft N03442. Hence, 242 passengers are recovered at Phase I of the algorithm. Due to the insufficient capacity of aircraft N03442, 9 passengers of flight 16 are spilled and stay overnight. Note that flight 16 belongs to different itineraries and corresponding passengers are not equally important considering their contributions to airline's profit. To clarify, consider a direct passenger in itinerary 16 and a connecting passenger in itinerary 16-5. If the later one is spilled, then the passenger not only misses the first flight but also cannot take place on the next flight as it departs from ORD. Therefore, lost revenue is higher for the case when a connecting passenger is spilled instead of a direct one. In this example, among all the passengers of flight 16, only 9 direct passengers are spilled.

Moreover, aircraft N27261 and N15425 are exchanged at ORD airport after the later one completes flight 4 . To put it another way, N15425 operates the second disrupted flight block, i.e. flights 17 and 18, whereas unavailable aircraft N27261 takes over flights 5 and 6. This means that flights 5 and 6 are cancelled. Again, due to the insufficient seat capacity of aircraft N15425, 1 passenger of flight 17 is spilled. Since there exist no alternative flights between ORD and MCO in either direction, passengers of flights 5 and 6 cannot be recovered and have to stay overnight. As the recovery is not possible, Integrated Search Algorithm enforces those flights to operate by fixing the assignment variables in the mathematical model with the aim of finding a better solution. That is, Phase I is solved with the following additional constraints in the next iteration: $\sum_{t \in T \backslash\left\{t^{*}\right\}} z_{5}^{t}=1$ and $\sum_{t \in T \backslash\left\{t^{*}\right\}} z_{6}^{t}=1$. In fact, solution of iteration 2 yields the highest profit value under that time limit. We give the time-space network for the proposed schedule in Fig. 2 (b).

In the proposed solution, again cruise speed control mechanism is used to recover first flight block. Different from the previous iteration, aircraft N27261 and N15438 are exchanged upon the completion of flight 8. Hence, flights 9 and 10 are cancelled. 7 passengers who are initially assigned to flight 9 between ORD-IAH are re-routed over DFW airport and determined to follow ORD-DFW-IAH path. As they arrive at their destination airport $105 \mathrm{~min}$ late and have to fly one additional flight, cost of delay and flying an extra flight are considered in the objective value as well. Besides, 24 passengers of flight 10 are reassigned to flight 14 , a late alternative. In total, 31 passengers are recovered at Phase II. Observe that ticket prices of the cancelled flights 9 and 10 are higher compared to the cancelled flights at the previous iteration. However, when the actual profit is calculated considering individual passenger itinerary based recovery decisions, overall profit is increased and number of overnight passengers are decreased. If the ticket price was the only measure for the profitability of the flight, this solution could not have been achieved.

In Table 3, profit values, passenger analysis and corresponding cost components are provided. Number of cancelled flights is decreased from 4 to 2 compared to the solution where disrupted flights are cancelled directly. However, this is not the only reason behind the increase in profit. The proposed algorithm allows to operate more profitable flights by exchange of aircraft. As well as the reduction in overnight cost, increase in revenue compensate for the increase in compression and passenger recovery costs. Therefore, the resulting schedule improves the airline's profit by $66.1 \%$ and decreases the number of overnight passengers by $59.2 \%$ in comparison with the base policy where all disrupted flights are directly cancelled.

\section{Computational results}

In this section, we test the performance of math-heuristic algorithm developed for integrated aircraft and passenger recovery problem. We propose different strategies by allowing certain recovery actions. We evaluate and compare the performance of these strategies with respect to solution quality under time limit. All computational experiments are performed on $2.4 \mathrm{GHz}$ Intel Core i7-4700HQ CPU with $16 \mathrm{~GB}$ memory. The proposed algorithm is implemented in Java with a connection to
IBM ILOG CPLEX Optimization Studio 12.6.2.

In order to generate a sample schedule for testing the practicality of our algorithm, flight information along with aircraft schedules is extracted from "Airline On-Time Performance" database, provided by Bureau of Transportation Statistics (BTS). Extracted data involve information regarding tail numbers, origin and destination airports, departure and arrival times. In this sample schedule, Chicago O'Hare International Airport (ORD) and Dallas/Fort Worth International Airport (DFW) serve as hub airports. It is generated in a way that all aircraft originate their first flight from these hub airports and revisit there at least once within the same day. It includes 208 flights which are operated by 53 different aircraft. 34 different airports are visited by these 208 flights. This schedule can be found in Appendix C. We consider disruption scenarios at which one aircraft is unavailable at a time. We assume that mechanical failures are detected and aircraft undergo maintenance at hub airports. Therefore, aircraft have to stay at hub airport during the period of unavailability. Whenever the technical problem is solved or the maintenance is completed, aircraft become ready to operate.

We consider six aircraft types each having different features. Number of seats, mass, wing surface area, MRC speed and base turntime for each type are displayed in Table 4. Depending on the historical data regarding fuel prices, we assume unit cost of fuel, $\mathrm{c}_{\text {fuel }}=1.2 \$ / \mathrm{kg}$ and unit cost of $\mathrm{CO}_{2}$ emission, $\mathrm{c}_{\mathrm{CO}_{2}}=0.02 \$ / \mathrm{kg}$ (IATA, 2019). We will also provide an analysis for different fuel cost settings in Section 5.2. We use the cruise stage fuel flow model proposed by the Base of Aircraft Data (BADA) to calculate the fuel burn as in Şafak et al. (2017). Turnaround times are calculated by multiplying the base turntime with the congestion coefficient of the destination airport as discussed in Duran et al. (2015). This means, the higher the congestion level is, the more time aircraft needs to spend on ground until it takes off again for a new flight.

We assume that passenger itineraries are composed of either a single flight leg or two legs. Each flight is included as a single-leg itinerary. Also, flight pairs are added to two-leg itineraries as long as the minimum passenger connection time is satisfied and the maximum connection time is not exceeded. We set all minimum and maximum connection times to $30 \mathrm{~min}$ and $6 \mathrm{~h}$ respectively. Here, it is important to mention that passengers do not necessarily change planes for connecting flights. Their first and second flight legs might be operated by the same aircraft. Moreover, itineraries do not necessarily start at a hub airport; instead, any airport can be a starting point. However, we assume that passengers can connect only over hub airports. For instance, a passenger who wants to travel from DEN to ROC needs to follow DEN-ORD-ROC path where ORD is the hub airport. We have generated 685 itineraries in total. Note that each aircraft has different number of seats depending on its type. First, number of passengers assigned to each flight is generated randomly considering seat capacities and $70 \%$ seat occupancy rate is ensured for each flight. Then, passengers in each flight are allocated randomly to the itineraries which involve that particular flight.

For ticket prices, origin and destination airports as well as the time of the day are two determinant factors. Base ticket prices are obtained from the report provided by U.S. Department of Transportation (2015). These values show average fares between certain origin and destination pairs, regardless of the departure time. In order to incorporate the time factor, we use the segmentation idea provided in pafak et al. (2018). As displayed in Table 5, we divide the day into three segments in descending

Table 4

Aircraft parameters.

\begin{tabular}{lllllll}
\hline Aircraft type & 1 & 2 & 3 & 4 & 5 & 6 \\
\hline Number of seats & 134 & 122 & 148 & 172 & 180 & 218 \\
Mass $(\mathrm{kg})$ & 74000 & 50000 & 61200 & 62000 & 64000 & 135000 \\
Surface $\left(m^{2}\right)$ & 157.9 & 105.4 & 118 & 122.4 & 122.6 & 283.3 \\
MRC speed $(\mathrm{km} / \mathrm{h})$ & 867.6 & 859.2 & 867.6 & 855.15 & 868.79 & 876.7 \\
Base turntime $(\mathrm{min})$ & 32 & 36 & 26 & 28 & 30 & 40 \\
\hline
\end{tabular}


order of preference to differentiate between peak and unfavorable times from the perspective of passengers. Hence, the highest fare is incurred for first segment and so on. We calculate the ticket prices by multiplying base fare with coefficients $1.2,1.0$ and 0.8 , respectively.

We work on networks where passenger itineraries and aircraft routings are superimposed. Therefore, it is significant to understand and model the passenger behaviour while applying recovery strategies. If a flight is cancelled and recovery is not possible, then passengers have to stay overnight at their origin airports. Also, due to the departure delays or re-routing actions, passengers might arrive at their destination airports late compared to their planned arrivals. We model passenger behaviour in cases of cancellation and delay by corresponding cost parameters. Both of the cost terms reflect the compensation for accommodation, meals and other essential needs. They can be interpreted as the measures of passenger inconvenience and enable penalization of loss of customer goodwill. As proposed in Marla et al. (2017), we let the overnight cost per passenger as $\$ 50$ and delay cost per minute per passenger as \$1.09 in our experiments. In Section 5.2, we will provide an analysis for higher overnight cost settings as well. We calculate spill cost by adding overnight cost and ticket price. Lastly, cost of flying an additional flight is set to $\$ 10$, a small value, to create a disincentive when direct alternatives are available as well.

\subsection{Recovery strategies}

In the case of a disruption, one feasible solution is to cancel all flights of the unavailable aircraft, yet it might not be the best possible approach. We consider this as the base policy and evaluate alternative approaches in comparison with this strategy. Exchange of aircraft and cruise time compression are tools that can be used for rescheduling. Possible recovery strategies which are constructed by combining these tools are listed in Table 6 . If a tool is marked as 1 , then this means that it is allowed to be used for that strategy and 0 otherwise.

Strategy 1 (Base Policy, S1): It gives an immediate feasible solution without changing the rest of the schedule. Only affected flights are removed from the operating flights set, $z_{i}^{t^{*}}=1 \forall i \in D$. No re-timing decisions are made, keeping the departures same as the original schedule. However, it could yield high operational costs and passenger inconvenience. Passengers could be re-routed but AOCC might miss the opportunity of recovery as well.

Strategy 2 (S2): Exchange of aircraft provides flexibility while determining the cancellation set. When cancellation is inevitable, airlines prefer to operate more "profitable" flights. In this study, ticket price is not the only measure for the profitability, different from the other approaches in literature. To clarify, consider two flights with equal number of passengers but different ticket prices. In the case of cancellation, lost revenue is higher for the one with higher price. However, one needs to check whether the disrupted passengers can be recovered or not as well. Profit generated from the recovered passengers might compensate for the difference between ticket prices. In such a case, the one with higher ticket price can be cancelled. One drawback of exchange is that, it might cause spill of passengers due to insufficient seat capacity. As a significant contribution, we are able to compare flights in terms of profitability by evaluating each passenger itinerary individually.

Strategy 3 (S3): Idle times might not be sufficient to accommodate disrupted flights into tight schedules. At that point, aircraft cruise speed can be adjusted to open up some time by shortening predetermined flight times. We assume that all aircraft fly at MRC speed and non-cruise

Table 5

Time intervals for the price segmentation.

\begin{tabular}{ll}
\hline Segment & Time Interval \\
\hline 1 & $06: 00-08: 59$ and after 17:00 \\
2 & $09: 00-11: 59$ and 15:00-16:59 \\
3 & $12: 00-14: 59$ \\
\hline
\end{tabular}

Table 6

Recovery strategies.

\begin{tabular}{lll}
\hline Strategy & Exchange $(0 / 1)$ & Compression $(0 / 1)$ \\
\hline S1 (Base Policy) & 0 & 0 \\
S2 & 1 & 0 \\
S3 & 0 & 1 \\
S4 & 1 & 1 \\
\hline
\end{tabular}

operations take $30 \mathrm{~min}$. We allow the compression of cruise times up to $15 \%$ due to technical limitations. Although it incurs additional fuel burn and $\mathrm{CO}_{2}$ emission cost, it is the best possible option in terms of passenger recovery. Unless there exist spilled passengers due to the lack of seat capacity, it enables AOCC to recover all passengers at once without considering alternative re-routing options. Maintaining the passenger convenience could easily compensate for additional fuel, $\mathrm{CO}_{2}$ emission and spill cost.

Strategy 4 (S4): It represents the combination of both tools. Therefore, it encompasses all the advantages listed under Strategies 2 and 3 . As well as the individual benefits, using them simultaneously is also favorable. If cruise times of flights are shortened by speeding up aircraft which are planned to land to hub airports, then these aircraft become available earlier than expected. This generates more exchange alternatives and enlarges the solution space, enhancing the flexibility over operations.

Unless indicated otherwise, we run our algorithm for $60 \mathrm{~s}$. We designate profit and the number of passengers who have to stay overnight as performance measures. We consider the base policy as a reference point and make comparisons accordingly. Improvement percentages for overnight passengers are calculated using the following formula:

Overnight Improvement $(\%)=100 \times \frac{\text { Overnight }_{\text {Base }}-\text { Overnight }_{\text {Proposed }}}{\text { Overnight }_{\text {Base }}}$

where Overnight $t_{\text {Base }}$ and Overnight Proposed $_{\text {denote the number of overnight }}$ passengers under base policy and the proposed strategy, respectively. For the improvement in terms of profit, we follow a similar logic with loss of profit values. If all aircraft were available, then airline would have reached its planned profit value, $P_{\text {Original }}$. In the case of disruption, a deviation occurs, but only a small portion of flights are subject to change. Rest of them remain as in the original schedule and hence their contribution to the objective value does not change. In order to exclude the impact of unaffected flights, we make our calculations with respect to the loss of profit values. When the base policy is applied, loss of profit can be calculated as $P_{\text {Original }}-P_{\text {Base }}$. Likewise, $P_{\text {Original }}-P_{\text {Proposed }}$ corresponds to the loss when the proposed strategy is implemented. Improvement percentages for marginal profits are calculated using the following formula:

Profit Improvement $(\%)=100 \times \frac{\text { Loss }_{\text {Base }}-\text { Loss }_{\text {Proposed }}}{\text { Loss }_{\text {Base }}}$

\subsection{Scenario analysis}

In order to observe which recovery tools work well under which circumstances, we take the schedule provided in Appendix as the original schedule and investigate four disruption cases. Related flights and the characteristics of the unavailability scenarios can be found in Tables 7 and 8 respectively. This table shows the unavailable aircraft, duration of the disruption and affected flights which are initially assigned to that unavailable aircraft. We generate 10 instances for each case by randomly generating number of passengers on each flight. For the first two cases, unavailability period is short in a sense that only one flight block is disrupted. For the other cases, aircraft is not able to serve throughout the day and two flight blocks are affected. In Case 1, it is apparent that flight 206 is disrupted since the aircraft cannot depart at 
Table 7

Flights which are related to disruption scenarios.

\begin{tabular}{lllllll}
\hline $\begin{array}{l}\text { Tail } \\
\text { No }\end{array}$ & $\begin{array}{l}\text { Flight } \\
\text { No }\end{array}$ & Departure & Arrival & $\begin{array}{l}\text { Departure } \\
\text { Time }\end{array}$ & $\begin{array}{l}\text { Flight } \\
\text { Time }\end{array}$ & $\begin{array}{l}\text { Arrival } \\
\text { Time }\end{array}$ \\
\hline N352 & 201 & ATL & ORD & $07: 30$ & $02: 20$ & $09: 50$ \\
N352 & 202 & ORD & BOS & $13: 45$ & $03: 00$ & $16: 45$ \\
N352 & 203 & BOS & ORD & $19: 30$ & $02: 50$ & $22: 20$ \\
\hline N251 & 204 & ORD & MSP & $09: 30$ & $01: 45$ & $11: 15$ \\
N251 & 205 & MSP & ORD & $12: 25$ & $01: 40$ & $14: 05$ \\
N251 & 206 & ORD & ROC & $16: 00$ & $02: 30$ & $18: 30$ \\
N251 & 207 & ROC & ORD & $19: 30$ & $02: 20$ & $21: 50$ \\
\hline
\end{tabular}

Table 8

Unavailability scenarios.

\begin{tabular}{llll}
\hline $\begin{array}{l}\text { Unavailability } \\
\text { Scenario }\end{array}$ & $\begin{array}{l}\text { Unavailable } \\
\text { Aircraft }\end{array}$ & $\begin{array}{l}\text { Unavailability } \\
\text { Duration }\end{array}$ & $\begin{array}{l}\text { Affected } \\
\text { Flights }\end{array}$ \\
\hline Case 1 & N251 & $16: 00-19: 00$ & $206 / 207$ \\
Case 2 & N251 & $09: 30-14: 00$ & $204 / 205$ \\
Case 3 & N251* & $09: 30-22: 00$ & $204 / 205 / 206 / 207$ \\
Case 4 & N251 & $09: 30-22: 00$ & $204 / 205 / 206 / 207$ \\
\hline
\end{tabular}

planned departure time. Even though the departure of the next flight, 207 , does not coincide with the unavailability period, it is also affected. This is because the aircraft cannot reach to the airport where flight 207 is supposed to depart. Therefore, it is indifferent if the unavailability period is between 16:00 and 19:00 or 16:00 and any time after 19:00. In Case 2, disruption period is still short but distinctly this flight block can fit into the existing schedule if cruise times are adjusted. Case 3 and 4 both involve the disrupted flight blocks which will be examined under first two cases. For the Case 3, we make a slight change in the original schedule as denoted with * in Table 8 . We shift the departure of flight 201 , which is assigned to aircraft N352, by $1 \mathrm{~h}$ so as to prevent accommodation of the first disrupted flight block into the schedule of that operating aircraft. Finally, Case 4 is intended to reflect a scenario where both exchange and compression tools have significant contributions to the objective.

We conduct computational experiments for all cases under four strategies. For each case, Tables 9-12 show characteristics of recovery plans generated by all strategies. Number of cancellations, number of recovered passengers at each phase, cost components and improvement percentages are listed. We take ten replications for each factor combination to observe if random values of passenger demand have any impact on objective values. Note that the values of Tables 9-12 are the average among 10 randomly generated instances. Using these strategies, we conduct what-if analysis to measure how changes in a set of recovery actions impact the profit and passenger convenience.

What if either cruise time compression or exchange of aircraft is not allowed? (Strategy 1, Base Policy) Since no re-timing decisions are made, departure times remain constant for the operating flights and passenger

Table 9

Comparison of different recovery strategies for Case 1 .

\begin{tabular}{lllll}
\hline & S1 & S2 & S3 & S4 \\
\hline \# of cancellations & 2 & 1 & 2 & 1 \\
\# of recovered passengers & 0 & 28 & 0 & 25 \\
$\rightarrow$ Phase I & 0 & 0 & 0 & 0 \\
$\rightarrow$ Phase II & 0 & 28 & 0 & 25 \\
\# of overnight passengers & 253 & 89 & 253 & 90 \\
\% Imp. Overnight & 0 & 64.8 & 0 & 64.4 \\
\hline Total compression (min) & 0 & 0 & 0 & 5 \\
Compression cost (\$) & 0 & 0 & 0 & 43 \\
Passenger delay cost (\$) & 0 & 0 & 0 & 310 \\
Overnight cost (\$) & 12650 & 8264 & 12650 & 6938 \\
Objective (\$) & 4605744 & 4645523 & 4605744 & 4645924 \\
\% Imp. Profit & 0 & 62.3 & 0 & 62.9 \\
\hline
\end{tabular}

Table 10

Comparison of different recovery strategies for Case 2 .

\begin{tabular}{lllll}
\hline & S1 & S2 & S3 & S4 \\
\hline \# of cancellations & 2 & 2 & 0 & 0 \\
\# of recovered passengers & 0 & 11 & 253 & 253 \\
$\rightarrow$ Phase I & 0 & 0 & 253 & 253 \\
$\rightarrow$ Phase II & 0 & 11 & 0 & 0 \\
\# of overnight passengers & 253 & 244 & 0 & 0 \\
\% Imp. Overnight & 0 & 3.6 & 100 & 100 \\
\hline Total compression (min) & 0 & 0 & 21 & 21 \\
Compression cost (\$) & 0 & 0 & 116 & 116 \\
Passenger delay cost (\$) & 0 & 2703 & 0 & 0 \\
Overnight cost (\$) & 12650 & 12215 & 0 & 0 \\
Objective (\$) & 4605744 & 4606599 & 4657777 & 4657777 \\
\% Imp. Profit & 0 & 1.6 & 98.1 & 98.1 \\
\hline
\end{tabular}

Table 11

Comparison of different recovery strategies for Case 3.

\begin{tabular}{lllll}
\hline & S1 & S2 & S3 & S4 \\
\hline \# of cancellations & 4 & 3 & 4 & 3 \\
\# of recovered passengers & 0 & 40 & 0 & 45 \\
$\rightarrow$ Phase I & 0 & 0 & 0 & 0 \\
$\rightarrow$ Phase II & 0 & 40 & 0 & 45 \\
\# of overnight passengers & 503 & 305 & 503 & 292 \\
\hline \% Imp. Overnight & 0 & 39.2 & 0 & 41.7 \\
Total compression (min) & 0 & 0 & 0 & 3 \\
Compression cost (\$) & 0 & 0 & 0 & 38 \\
Passenger delay cost (\$) & 0 & 3921 & 0 & 4070 \\
Overnight cost (\$) & 25155 & 16585 & 25155 & 16154 \\
Objective (\$) & 4593239 & 4634544 & 4593239 & 4636933 \\
\% Imp. Profit & 0 & 35.1 & 0 & 37.1 \\
\hline
\end{tabular}

Table 12

Comparison of different recovery strategies for Case 4.

\begin{tabular}{lllll}
\hline & S1 & S2 & S3 & S4 \\
\hline \# of cancellations & 4 & 2.4 & 2 & 1 \\
\# of recovered passengers & 0 & 41 & 253 & 278 \\
$\rightarrow$ Phase I & 0 & 0 & 253 & 253 \\
$\rightarrow$ Phase II & 0 & 41 & 0 & 25 \\
\# of overnight passengers & 503 & 265 & 250 & 88 \\
\hline \% Imp. Overnight & 0 & 47.2 & 50.3 & 82.6 \\
Total compression (min) & 0 & 0 & 21 & 30 \\
Compression cost (\$) & 0 & 0 & 116 & 209 \\
Passenger delay cost (\$) & 0 & 8557 & 0 & 1442 \\
Overnight cost (\$) & 25155 & 14392 & 12505 & 5420 \\
Objective (\$) & 4593239 & 4646106 & 4645274 & 4685100 \\
\% Imp. Profit & 0 & 45.1 & 44.6 & 78.8 \\
\hline
\end{tabular}

delay cost is zero. Additional fuel and $\mathrm{CO}_{2}$ emission cost is not incurred as well. In all cases, S1 yields the lowest profit along with highest overnight cost. Therefore, it should be considered as a last resort.

What if only exchange of aircraft is allowed? (Strategy 2) It has a significant effect on the number of cancellations, overnight passengers and profit. In recovery solutions of Case 1 and 3, number of cancellations are decreased by one when exchange is allowed. This is because the algorithm prefers to cancel one long haul flight instead of cancelling two short haul flights. This action leads to a considerable decrease in number of overnight passengers along with an increase in profit value. Another observation is that number of passengers who are recovered at Phase II are increased as well. The model makes cancellations in a way that the solution set creates more re-accommodation options for passengers.

What if only cruise time compression is allowed? (Strategy 3) It allows us to accommodate disrupted flight blocks into the existing schedule by shortening cruise times. In Case 2, all disrupted passengers are recovered at the same time, leading to great savings from overnight cost. As all the 
affected passengers are recovered at once, there is no need for reaccommodation. Compared to the results of S1, number of overnight passengers are decreased by $100 \%$ whereas the profit is improved by 98.1\%. Compression cost which stands for the additional fuel and $\mathrm{CO}_{2}$ emission is even negligible compared to the increase in profit. Same impact is observed in Case 4 when compression tool is allowed to be used. Hence, it is highly preferable in terms of passenger recovery.

What if exchange of aircraft and cruise time compression are both allowed? (Strategy 4) It is beneficial in terms of generating new exchange options. For instance, in Case 1, when S3 is applied, there is no change in the solution compared to $\mathrm{S} 1$ and none of the cruise times are compressed. This is because it is not possible to place a disrupted block into the schedule by shortening flight times. So it is not meaningful to incur additional fuel and $\mathrm{CO}_{2}$ emission costs. Aircraft continue to operate at MRC speed. However when S4 is used, it is observed that the model makes a compression of $5 \mathrm{~min}$. Moreover, difference between the results of S2 and S4 indicates that unavailable aircraft is exchanged with another aircraft when S4 is applied, otherwise objectives would be the same for both strategies. Therefore, we can conclude that the model prefers to shorten flight time to generate a new exchange option. A similar observation holds for Case 4 as well.

In Tables 9-12, total delay cost depends on the number of passengers who are recovered at Phase II. They are either assigned to late alternatives or fly an extra flight and reach destination airports later than planned. However in Case 1 under S2, 28 passengers are recovered without incurring any delay cost. This indicates that they are connecting passengers who are rerouted to early alternatives in the second leg of their connections. As they are notified in advance of their continuing flight, airline is not penalized.

Another observation is that, in Case 1, although the number of overnight passengers is increased, profit is higher for S4 compared to S2. This shows that increase in profit does not necessarily imply the decrease in overnight passengers. Here, profitability of passengers surpasses the quantity.

Unless real-time and efficient recovery solutions are available, unavailability of aircraft might result in loss of customer goodwill and damage the profit of airline substantially. In Table 13, \% improvement of profits are compared under different time limits: 45 and $60 \mathrm{~s}$. We observe that even after $45 \mathrm{~s}$, the results remain same except for a few strategies. Therefore, we do not feel the need to extend solution time beyond $60 \mathrm{~s}$. Moreover, 10 replications are performed for each case and strategy to see whether random number of passengers have any effect on objective values. Minimum, maximum and average improvement percentages under different time limits are provided in Table 13.

Among all strategies, it is evident that Strategy 4 dominates the rest of the policies. Both exchange and compression tools enable our model

Table 13

$\%$ Improvement of the profit under different time limits.

\begin{tabular}{|c|c|c|c|c|c|c|c|}
\hline & & \multicolumn{3}{|l|}{$45 \mathrm{~s}$} & \multicolumn{3}{|l|}{$60 \mathrm{~s}$} \\
\hline & & $\min$ & avg & $\max$ & $\min$ & avg & $\max$ \\
\hline \multirow[t]{4}{*}{ Case 1} & $\mathrm{~S} 1$ & - & - & - & - & - & - \\
\hline & $\mathrm{S} 2$ & 51.8 & 62.3 & 75.9 & 51.8 & 62.3 & 75.9 \\
\hline & S3 & 0 & 0 & 0 & 0 & 0 & 0 \\
\hline & S4 & 54.4 & 62.9 & 75.9 & 54.4 & 62.9 & 75.9 \\
\hline \multirow[t]{4}{*}{ Case 2} & $\mathrm{~S} 1$ & - & - & - & - & - & - \\
\hline & $\mathrm{S} 2$ & 0 & 1.6 & 15.3 & 0 & 1.6 & 15.3 \\
\hline & S3 & 97.6 & 98.1 & 98.3 & 97.6 & 98.1 & 98.3 \\
\hline & S4 & 97.6 & 98.1 & 98.3 & 97.6 & 98.1 & 98.3 \\
\hline \multirow[t]{4}{*}{ Case 3} & $\mathrm{~S} 1$ & - & - & - & - & - & - \\
\hline & S2 & 17 & 34.5 & 44.6 & 21.3 & 35.1 & 44.6 \\
\hline & S3 & 0 & 0 & 0 & 0 & 0 & 0 \\
\hline & S4 & 2.7 & 32.7 & 44.6 & 22.4 & 37.1 & 44.6 \\
\hline \multirow[t]{4}{*}{ Case 4} & $\mathrm{~S} 1$ & - & - & - & - & - & - \\
\hline & S2 & 26 & 42.9 & 49.9 & 26 & 45.1 & 61.6 \\
\hline & S3 & 39.4 & 44.6 & 51.2 & 39.4 & 44.6 & 51.2 \\
\hline & S4 & 71.5 & 78.8 & 91.8 & 71.5 & 78.8 & 91.8 \\
\hline
\end{tabular}

to achieve better results but when they are allowed simultaneously. \% improvement of overnight passengers and profit are considerably higher in Case 4. Therefore, we can propose airlines to use S4 for this integrated recovery problem.

What If Analysis on Overnight Cost: Due to the high level of competition in airline industry, we put a special emphasis on passenger convenience. Therefore, we perform an experimental design and run our algorithm with S4 for three overnight cost settings. For low (L), medium (M) and high $(\mathrm{H})$ values, we set the overnight cost per passenger to $50 \$$, $150 \$$ and 250\$, respectively. As depicted in Fig. 3, number of overnight passengers are decreased when overnight cost increases as expected.

Moreover, an increase in the overnight cost would result in a tradeoff between different cost terms. As the overnight cost increases, the model would have higher tendency to operate more flights and recover more passengers to prevent passengers from staying overnight. Increasing the cruise speed of a flight directly reduces its block time, and thereby opening up space to accommodate disrupted flights into the flight schedule. Although cruise time reduction provides a great opportunity to add more flights, increasing the speed of an aircraft comes with significant additional cost of fuel burn. Therefore, higher overnight costs might yield higher fuel costs. Besides, delaying a passenger becomes a more preferable option compared to making her stay overnight. Some passengers, who supposed to stay overnight, might be recovered by facing longer delays.

Fuel cost has the major component of overall operational expenses. Therefore, it has the highest impact on the profit compared to other cost terms. For instance, an increase in the fuel cost results in a significant drop in profit values as can be seen from Fig. 4. Although this effect dominates Fig. 4, profit values under S4 still change if the overnight cost is $\mathrm{L}, \mathrm{M}$, or $\mathrm{H}$ for a fixed level of fuel cost. Table 14 represents how profit varies between three overnight cost settings for a given level of fuel cost for Case 3.

What If Analysis on Fuel Cost: Fuel cost, which is a significant portion of the total expenditure of airline operations, is highly variable. Therefore, in addition to the overnight cost per passenger, we take the fuel cost as an experimental factor. We set the unit cost of fuel to 0.6, 1.2 and $1.8 \$ / \mathrm{kg}$ for low (L), medium (M) and high $(\mathrm{H})$ values. For each case and factor level combination, we run our algorithm with S4 and S1. Here, the difference between S4 and S1 can be interpreted as the value of our proposed math-heuristic algorithm. It shows how much we can improve the profit by allowing exchange of aircraft and cruise time compression instead of directly cancelling all affected flights.

As expected, an increase in overnight or fuel cost results in a lower profit value. A significant observation is that the difference between the profit values of S4 and S1 increases as the overnight cost increases in all cases, regardless of the fuel cost level. That is, the value of our algorithm is higher and it achieves better savings when the overnight cost is high. For instance, Fig. 4 represents how this difference varies between three overnight cost settings for Case 4. As can be observed from this figure, the largest difference is obtained when both factors are set to their highest values. Moreover, for a fixed overnight cost level, when the fuel cost increases, difference values might slightly decrease as in Case 4. However, they are still considerable and applying S4 contributes to the profit of the airline in a substantial manner. For the other cases, the results follow the same trend as well.

\section{Conclusion and future work}

We focus on disruption scenarios at which aircraft become unavailable due to mechanical failures or extension on maintenance durations. We intend to develop a structural framework to aid airlines in handling disruptions at operational level. To this end, we address aircraft and passenger recovery problems simultaneously and propose a novel mathheuristic algorithm to solve it. We aim to come up with a recovery plan which maximizes the profit of airline while also mitigating the passenger dissatisfaction. 
Case 1

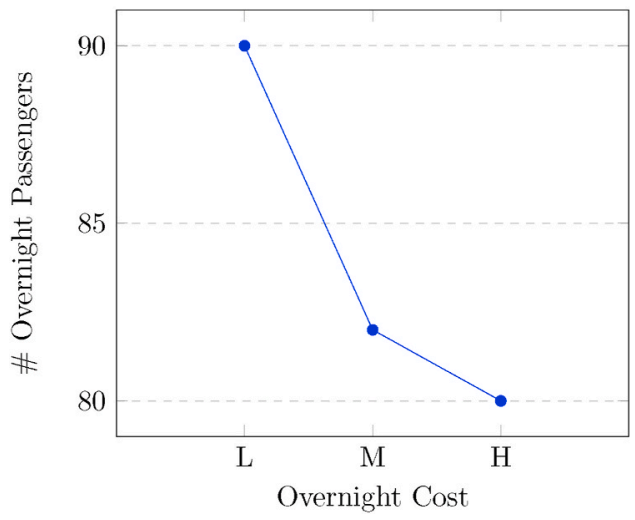

Case 3

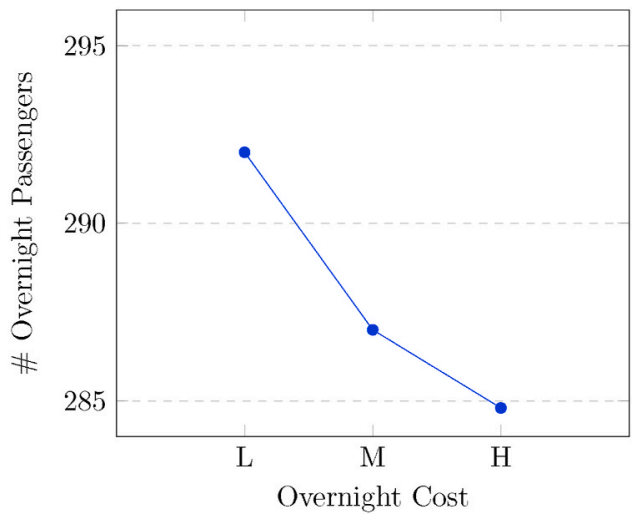

Fig. 3. Number of overnight passengers for different overnight cost settings.

Overnight Cost: L

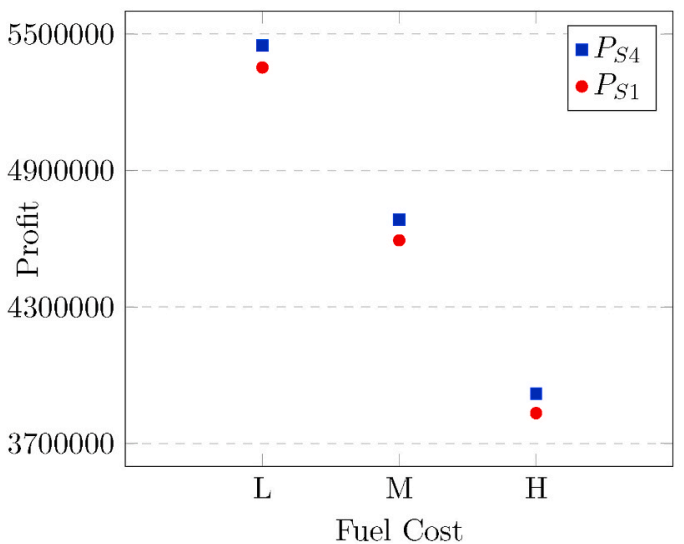

Overnight Cost: M

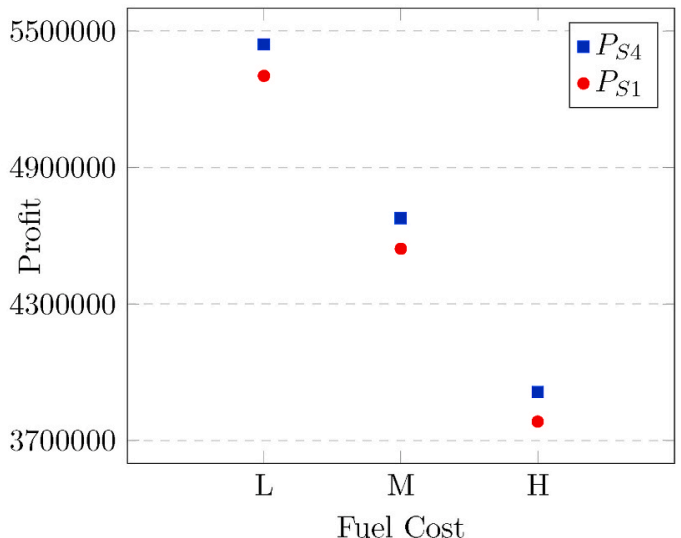

Overnight Cost: $\mathrm{H}$

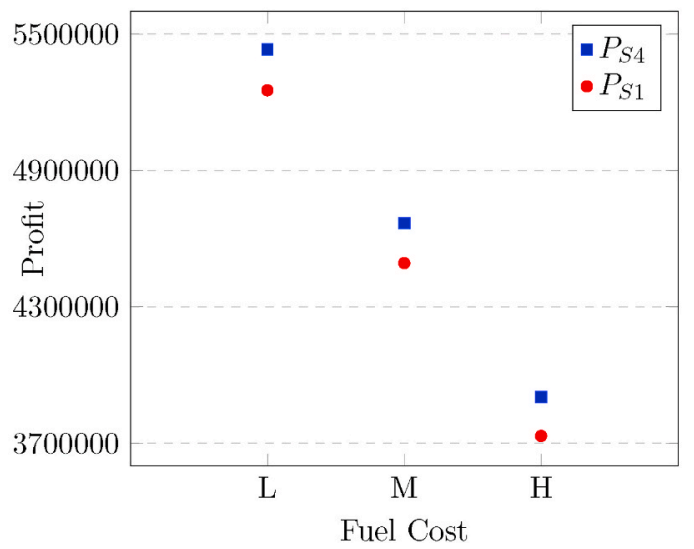

Fig. 4. What-if analysis on fuel cost for Case 4.

Table 14

Profit values of different factor level combinations for Case 3.

\begin{tabular}{llll}
\hline \multirow{2}{*}{ Fuel Cost } & \multicolumn{2}{l}{ Overnight Cost } & H \\
\cline { 2 - 4 } & L & M & 5337492 \\
\hline L & 5395792 & 5367442 & 4581161 \\
M & 4636933 & 4610141 & 3824111 \\
H & 3875297 & 3852841 & \\
\hline
\end{tabular}

Given the unavailable aircraft and disruption duration, our algorithm proceeds in two phases. In the first phase, we propose a nonlinear mixed integer recovery optimization model and adopt a conic quadratic optimization approach to mitigate computational difficulty as a theoretical contribution of this study. We allow changes in fleet type assignment by using exchange of aircraft as a recovery tool. Moreover, we provide the flexibility of speeding up aircraft to shorten cruise times. Lastly, if the recovery is not possible by any means, then cancellation option can be utilized. In the second phase, we devise an itinerary-based passenger recovery algorithm which evaluates each itinerary separately. 
As long as there exist available seats, passengers are either reaccommodated to alternative flights or rerouted over another hub airport. The integration between these phases is maintained by the integrated search algorithm through variable fixing. Ferrying an available aircraft or utilization of reserve aircraft can also be included to the set of recovery tools as an extension to this study.

We make recovery decisions based on passenger itineraries as a significant contribution. This allow us to re-accommodate passengers who are initially assigned to the same flight to different paths. Moreover, we prioritize itineraries with respect to their contributions to the objective value. This enables us to use limited resources, in this case available seats, in a better way. It is common for airlines to privilege first-class passengers or important passengers over others to ensure passenger satisfaction and loyalty. In addition to the distinction based on itineraries, fare class can also be a determinant factor while distinguishing the relative importance of each passenger. A single leg firstclass passenger could contribute more to the profit of the airline that a two-leg leisure itinerary. In that case, we would prioritize them as opposed to connecting passengers. Therefore, economy and business class passengers can be evaluated separately in further studies.

We work on networks where aircraft routings and passenger itineraries are superimposed. As being an integrated approach, our algorithm captures the dependencies between aircraft and passenger schedules. By taking these dependencies into the account, we provide the actual cancellation cost formulations. This is another crucial contribution of our research since passenger convenience is of high importance for airlines. In this regard, we manage to find the optimal trade-off between operating and passenger-related costs.

This integrated model can be used as a practical tool when airlines face unscheduled maintenance disruptions arising from contract negotiations or when some aircraft are grounded as a safety precaution. Under such disruption scenarios, it provides real time and efficient recovery solutions to avoid substantial loss of profit as well as maintaining customer satisfaction.

\section{Acknowledgement}

The authors thank the editor-in-chief and four anonymous referees for their constructive comments and suggestions that significantly improved this paper. This research was partially supported by Scientific and Technological Research Council of Turkey (TUBITAK) under Grant $116 \mathrm{M} 542$.

\section{Appendix A. Supplementary data}

Supplementary data to this article can be found online at https://doi. org/10.1016/j.jairtraman.2020.101991.

\section{References}

Abdelghany, K.F., Abdelghany, A.F., Ekollu, G., 2008. An integrated decision support tool for airlines schedule recovery during irregular operations. Eur. J. Oper. Res. 185, 825-848. https://doi.org/10.1016/j.ejor.2006.12.045.

Aktürk, M.S., Atamtürk, A., Gürel, S., 2014. Aircraft rescheduling with cruise speed control. Oper. Res. 62, 829-845. https://doi.org/10.1287/opre.2014.1279.

Aktürk, M.S., Atamtürk, A., Gürel, S., 2009. A strong conic quadratic reformulation for machine-job assignment with controllable processing times. Oper. Res. Lett. 37, 187-191. https://doi.org/10.1016/j.orl.2008.12.009.

Aloulou, M.A., Haouari, M., Zeghal Mansour, F., 2013. A model for enhancing robustness of aircraft and passenger connections. Transport. Res. C Emerg. Technol. 32, 48-60. https://doi.org/10.1016/j.trc.2013.03.008.

Arikan, U., Gürel, S., Aktürk, M.S., 2017. Flight network-based approach for integrated airline recovery with cruise speed control. Transport. Sci. 51, 1259-1287. https:// doi.org/10.1287/trsc.2016.0716.
Ben Ahmed, M., Ghroubi, W., Haouari, M., Sherali, H.D., 2017. A hybrid optimizationsimulation approach for robust weekly aircraft routing and retiming. Transport. Res. C Emerg. Technol. 84, 1-20. https://doi.org/10.1016/j.trc.2017.07.010.

Ben Ahmed, M., Zeghal Mansour, F., Haouari, M., 2018. Robust integrated maintenance aircraft routing and crew pairing. J. Air Transport. Manag. 73, 15-31. https://doi. org/10.1016/j.jairtraman.2018.07.007.

Bisaillon, S., Cordeau, J.F., Laporte, G., Pasin, F., 2011. A large neighbourhood search heuristic for the aircraft and passenger recovery problem. 4OR 9, 139-157. https:// doi.org/10.1007/s10288-010-0145-5.

Bratu, S., Barnhart, C., 2006. Flight operations recovery: new approaches considering passenger recovery. J. Sched. 9, 279-298. https://doi.org/10.1007/s10951-0066781-0.

Clausen, J., Larsen, A., Larsen, J., Rezanova, N.J., 2010. Disruption management in the airline industry - concepts, models and methods. Comput. Oper. Res. 37, 809-821. https://doi.org/10.1016/j.cor.2009.03.027.

Cook, A., Tanner, G., Williams, V., Meise, G., 2009. Dynamic cost indexing - managing airline delay costs. J. Air Transport. Manag. 15, 26-35. https://doi.org/10.1016/j. jairtraman.2008.07.001.

Duran, A.S., Gürel, S., Aktürk, M.S., 2015. Robust airline scheduling with controllable cruise times and chance constraints. IIE Trans. 47, 64-83. https://doi.org/10.1080/ 0740817X.2014.916457.

Eurocontrol, 2012. User Manual for the Base of Aircraft Data (Bada) Revision 3.10. Tech, Rep. 12/04/10-45, EEC Technical/Scientific, Eurocontrol. Eurocontrol Experimental Centre, France, 15, F-91222 Bretigny-sur-Orge.

Gürkan, H., Gürel, S., Aktürk, M.S., 2016. An integrated approach for airline scheduling, aircraft fleeting and routing with cruise speed control. Transport. Res. C Emerg. Technol. 68, 38-57. https://doi.org/10.1016/j.trc.2016.03.002.

IATA, 2019. Fuel price analysis. Available at: http://www.iata.org/publications/ economics/fuel-monitor/Pages/price-analysis.aspx. accessed May 2019.

Jafari, N., Zegordi, S.H., 2010. The airline perturbation problem: considering disrupted passengers. Transport. Plann. Technol. 33, 203-220. https://doi.org/10.1080/ 03081061003643788.

Jamili, A., 2017. A robust mathematical model and heuristic algorithms for integrated aircraft routing and scheduling, with consideration of fleet assignment problem. J. Air Transport. Manag. 58, 21-30. https://doi.org/10.1016/j. jairtraman.2016.08.008.

Jarrah, A.I.Z., Yu, G., Krishnamurthy, N., Rakshit, A., 1993. Decision support framework for airline flight cancellations and delays. Transport. Sci. 27, 266-280. https://doi. org/10.1287/trsc.27.3.266.

Kenan, N., Jebali, A., Diabat, A., 2018. The integrated aircraft routing problem with optional flights and delay considerations. Transport. Res. Part E Logist. Transp. Rev. 118, 355-375. https://doi.org/10.1016/j.tre.2018.08.002.

Khaled, O., Minoux, M., Mousseau, V., Michel, S., Ceugniet, X., 2018. A multi-criteria repair/recovery framework for the tail assignment problem in airlines. J. Air Transport. Manag. 68, 137-151. https://doi.org/10.1016/j.jairtraman.2017.10.002.

Kohl, N., Larsen, A., Larsen, J., Ross, A., Tiourine, S., 2007. Airline disruption management - perspectives, experiences and outlook. J. Air Transport. Manag. 13, 149-162. https://doi.org/10.1016/j.jairtraman.2007.01.001.

Lan, S., Clarke, J.P., Barnhart, C., 2006. Planning for robust airline operations: optimizing aircraft routings and flight departure times to minimize passenger disruptions. Transport. Sci. 40, 15-28. https://doi.org/10.1287/trsc.1050.0134.

Maher, S.J., 2015. A novel passenger recovery approach for the integrated airline recovery problem. Comput. Oper. Res. 57, 123-137. https://doi.org/10.1016/j. cor.2014.11.005.

Marla, L., Vaaben, B., Barnhart, C., 2017. Integrated disruption management and flight planning to trade off delays and fuel burn. Transport. Sci. 51, 88-111. https://doi. org $/ 10.1287 /$ trsc.2015.0609.

Marla, L., Vaze, V., Barnhart, C., 2018. Robust optimization: lessons learned from aircraft routing. Comput. Oper. Res. 98, 165-184. https://doi.org/10.1016/j. cor.2018.04.011.

Montlaur, A., Delgado, L., 2020. Flight and passenger efficiency-fairness trade-off for ATFM delay assignment. J. Air Transport. Manag. 83, 101758 https://doi.org/ 10.1016/j.jairtraman.2019.101758.

Petersen, J.D., Sölveling, G., Clarke, J.P., Johnson, E.L., Shebalov, S., 2012. An optimization approach to airline integrated recovery. Transport. Sci. 46, 482-500. https://doi.org/10.1287/trsc.1120.0414.

Rosenberger, J.M., Johnson, E.L., Nemhauser, G.L., 2003. Rerouting aircraft for airline recovery. Transport. Sci. 37, 408-421. https://doi.org/10.1287/ trsc.37.4.408.23271.

Şafak, Ö., Gürel, S., Aktürk, M.S., 2017. Integrated aircraft-path assignment and robust schedule design with cruise speed control. Comput. Oper. Res. 84, 127-145. https:// doi.org/10.1016/j.cor.2017.03.005.

Şafak, Ö., Çavuş, Ö., Aktürk, M.S., 2018. Multi-stage airline scheduling problem with stochastic passenger demand and non-cruise times. Transp. Res. Part B Methodol. 114, 39-67. https://doi.org/10.1016/j.trb.2018.05.012.

Sinclair, K., Cordeau, J.F., Laporte, G., 2014. Improvements to a large neighborhood search heuristic for an integrated aircraft and passenger recovery problem. Eur. J. Oper. Res. 233, 234-245. https://doi.org/10.1016/j.ejor.2013.08.034. 
Teodorovic, D., Guberinic, S., 1984. Optimal dispatching strategy on an airline network after a schedule perturbation. Eur. J. Oper. Res. 15, 178-182. https://doi.org/ 10.1016/0377-2217(84)90207-8.

U.S. Department of Transportation, 2015. Domestic airline consumer airfare report. Available at: https://data.transportation.gov/Aviation/Consumer-Airfare-Report-Ta ble-1a-All-U-S-Airport-P/tfrh-tu9e. accessed May 2019.

U.S. Department of Transportation, Federal Aviation Administration, 2019. FAA updates on boeing 737 MAX. Available at: https://www.faa.gov/news/updates/?news Id=93206. accessed May 2019.
Vink, J., Santos, B.F., Verhagen, W.J.C., Medeiros, I., Filho, R., 2020. Dynamic aircraft recovery problem - an operational decision support framework. Comput. Oper. Res. 117, 104892 https://doi.org/10.1016/j.cor.2020.104892.

Xiong, J., Hansen, M., 2013. Modelling airline flight cancellation decisions. Transport. Res. Part E Logist. Transp. Rev. 56, 64-80. https://doi.org/10.1016/j. tre.2013.05.003.

Zhang, D., Yu, C., Desai, J., Lau, H.Y.K.H., 2016. A math-heuristic algorithm for the integrated air service recovery. Transp. Res. Part B Methodol. 84, 211-236. https:// doi.org/10.1016/j.trb.2015.11.016. 